\title{
DCAMP: DISTRIBUTED COMMON API FOR MEASURING PERFORMANCE
}

\author{
A Thesis \\ presented to \\ the Faculty of California Polytechnic State University \\ San Luis Obispo
}

\author{
In Partial Fulfillment \\ of the Requirements for the Degree \\ Master of Science in Computer Science
}

by

Alexander Paul Sideropoulos

December 2014 
(C) 2014

Alexander Paul Sideropoulos

ALL RIGHTS RESERVED 


\section{COMMITTEE MEMBERSHIP}

TITLE:

dCAMP: Distributed Common API for Measuring Performance

AUTHOR: $\quad$ Alexander Paul Sideropoulos

DATE SUBMITTED: $\quad$ December 2014

COMMITTEE CHAIR: Michael Haungs, Ph.D. Associate Professor of Computer Science

COMMITTEE MEMBER: Aaron Keen, Ph.D.

Assistant Professor of Computer Science

COMMITTEe MEMBER: John Bellardo, Ph.D.

Associate Professor of Computer Science 


\section{ABSTRACT \\ $d C A M P$ : Distributed Common API for Measuring Performance}

\section{Alexander Paul Sideropoulos}

Although the nearing end of Moore's Law has been predicted numerous times in the past [22], it will eventually come to pass. In forethought of this, many modern computing systems have become increasingly complex, distributed, and parallel. As software is developed on and for these complex systems, a common API is necessary for gathering vital performance related metrics while remaining transparent to the user, both in terms of system impact and ease of use.

Several distributed performance monitoring and testing systems have been proposed and implemented by both research and commercial institutions. However, most of these systems do not meet several fundamental criterion for a truly useful distributed performance monitoring system: 1) variable data delivery models, 2) security, 3) scalability, 4) transparency, 5) completeness, 6) validity, and 7) portability [30].

This work presents $d C A M P$ : Distributed Common API for Measuring Performance, a distributed performance framework built on top of Mark Gabel and Michael Haungs' work with CAMP. This work also presents an updated and extended set of criterion for evaluating distributed performance frameworks and uses these to evaluate $d C A M P$ and several related works. 


\section{ACKNOWLEDGMENTS}

In no small part, this thesis is the result of the continuous support I have received from my family and friends throughout the process. I would also like to say a special thanks to my committee members and especially to my relentless advisor, Dr. Michael Haungs, who stuck with this project across all the years.

Lastly, I would like to express very special thanks to my beautiful bride,

Jessica Lindsay, for always encouraging me in this endeavor and selflessly giving of her time and energy that I might master this work for Father's glory. 


\section{TABLE OF CONTENTS}

LIST OF TABLES IX ix

LIST OF FIGURES I X

CHAPTERS

1 Introduction 1

1.1 Distributed Performance Framework Criterion . . . . . . . . . . . 2

1.1.1 Data Delivery Models . . . . . . . . . . . . . 2

1.1.2 Security ........................ 2

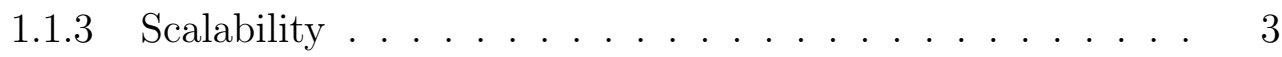

1.1.4 Transparency .................. . . 3

1.1.5 Completeness .................. . . 3

1.1.6 Validity ................... 4

1.1.7 Portability.................. 4

$1.2 \quad d C A M P \ldots \ldots \ldots \ldots \ldots \ldots$

1.2.1 Terminology . . . . . . . . . . . . . . 5 5

2 Design 9

2.1 Architecture ........................ 9

2.2 Requirements . . . . . . . . . . . . . . . . . 10

2.2.1 Functional ................... 10

2.2 .2 Non-Functional . . . . . . . . . . . . . . . 11

$2.3 d C A M P$ Roles and Services . . . . . . . . . . . . . . . 11

2.3.1 Services . . . . . . . . . . . . . . 11

2.3.2 Roles . . . . . . . . . . . . . . . . . . . 12

2.4 Fault Tolerance . . . . . . . . . . . . . . . . . . . . . . . 14

2.4.1 Heartbeating (Detecting Disconnections) . . . . . . . . 14

2.4.2 Reminder Algorithm (Metric Node Recovery) . . . . . . . 15

2.4.2.1 Detection ............... 16 
2.4.3 Promotion Algorithm (Collector Node Recovery) . . . . . 16

2.4.3.1 Detection . . . . . . . . . . . . 16

2.4.4 Election Algorithm (Root Node Recovery) . . . . . . . . 17

2.4.4.1 Detection . . . . . . . . . . . . . 18

$2.5 \quad d C A M P$ Metrics $\ldots \ldots \ldots \ldots \ldots \ldots$

2.5.1 Global Metrics . . . . . . . . . . . . . . . . . 18

2.5 .2 Network I/O Metrics . . . . . . . . . . . . . . . . 19

2.5 .3 Disk I/O Metrics . . . . . . . . . . . . . . . . . . . 19

2.5.4 Per-process Metrics . . . . . . . . . . . . . . . . . . . 19

2.5 .5 Inquiry Metrics . . . . . . . . . . . . . . 20

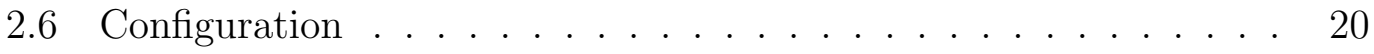

2.6.1 Node Specification . . . . . . . . . . . . . . . . . 21

2.6.2 Sample Specification . . . . . . . . . . . . . . . 22

2.6.2.1 Accumulative Time-Based Filtering Pitfall . . . . 22

3 Implementation 24

$3.1 d C A M P$ Operation . . . . . . . . . . . . . . . . 24

3.1.1 Sequence of $d C A M P$ Operation . . . . . . . . . . . . 24

3.1 .2 Threading Model . . . . . . . . . . . . . . . . 25

3.2 ZeroMQ Protocols . . . . . . . . . . . . . . . . 27

3.2 .1 Topology Protocols . . . . . . . . . . . . . . . . . . 27

3.2.1.1 Message Definitions _. . . . . . . . . . 28

3.2.2 Configuration Replication Protocol . . . . . . . . . . . . 29

3.2.2.1 Message Definitions _. . . . . . . . . . . 31

3.2 .3 Data Flow Protocol . . . . . . . . . . . . . . . . . 34

3.2.3.1 Performance Measurement . . . . . . . . . . . 35

3.2.3.2 Message Definitions . . . . . . . . . . . 36

3.2 .4 Recovery Protocols . . . . . . . . . . . . . . . . . 37

4 Analysis 40

4.1 Transparency . . . . . . . . . . . . . . . . . . . . 40

4.1 .1 Workload . . . . . . . . . . . . . . . 41

$4.1 .2 \quad$ dCAMP Configuration . . . . . . . . . . . . . . 42

4.1 .3 Results . . . . . . . . . . . . . . . . . . 43

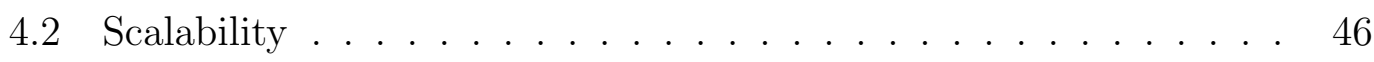

4.2 .1 Workload . . . . . . . . . . . . . . . . 46

$4.2 .2 \quad$ dCAMP Configuration . . . . . . . . . . . . . . 46

4.2 .3 Results . . . . . . . . . . . . . . . 47 
5 Related Work $\quad 51$

5.1 Analysis ....................... 51

5.1 .1 NetLogger .................. 52

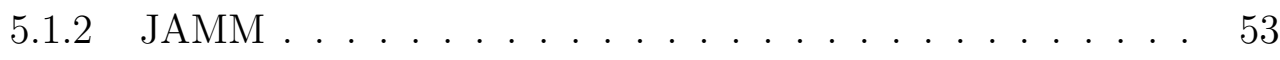

5.1 .3 Hawkeye . . . . . . . . . . . . . . . . 53

5.1 .4 SCALEA-G ..................... 54

5.1 .5 IMPuLSE . . . . . . . . . . . . . . . . . . 55

5.1 .6 Host sFlow . . . . . . . . . . . . . . 55

5.1 .7 Ganglia . . . . . . . . . . . . . . . 56

5.2 Summary . . . . . . . . . . . . . . . . . 57

6 Conclusions $\quad 58$

6.1 Summary of Contributions . . . . . . . . . . . . . . 58

6.2 Future Work . . . . . . . . . . . . . . . . 59

6.2.1 Additional Features . . . . . . . . . . . . . 59

6.2.2 Fault Tolerance . . . . . . . . . . . . . . . 60

6.2.3 Improve Performance and Scalability . . . . . . . . . . 61

6.2.4 Metric Extensions . . . . . . . . . . . . 62

$\begin{array}{ll}\text { BIBLIOGRAPHY } & 64\end{array}$

\section{APPENDICES}

$\begin{array}{lll}\text { A ZeroMQ Primer } & 68\end{array}$

A.1 Why ZeroMQ . . . . . . . . . . . . . . . . 68

A.2 Sockets and Message Patterns . . . . . . . . . . . . . . . 69

A.2.1 Sockets and Messages . . . . . . . . . . . . . 69

A.2.2 Messaging Patterns . . . . . . . . . . . . . . 70

A.3 Useful Features for $d C A M P \ldots \ldots$. . . . . . . . . . 71

A.3.1 Topic Filtering . . . . . . . . . . . . 71

A.3.2 Easy Message Debugging . . . . . . . . . . . . . . . 72

A.3.3 Simplified Threading Design . . . . . . . . . . . . 72

A.3.4 Quick Simulation .............. 72

B Real Life $\quad 74$ 


\section{LIST OF TABLES}

2.1 Role to Service Mappings . . . . . . . . . . . . . . . . . . . . 13

3.1 Metric Types . . . . . . . . . . . . . . . . 36 


\section{LIST OF FIGURES}

2.1 Configuration File - Node Specification . . . . . . . . . . . 21

2.2 Configuration File - Sample Specification . . . . . . . . . . . . . . 22

3.1 Sample Watchdog Script . . . . . . . . . . . . . . . . . . 24

3.2 Node, Role, Services Threading Model Diagram . . . . . . . . . . 26

3.3 Topology Protocols . . . . . . . . . . . . . . . . . . . . . . . . 27

3.4 Topology Protocol Diagram . . . . . . . . . . . . . 28

3.5 TOPO Message Definition . . . . . . . . . . . . . . 28

3.6 CONTROL Message Definition . . . . . . . . . . . . . . . . . . . 29

3.7 WTF Message Definition . . . . . . . . . . . . . . . . . . . . . 29

3.8 Configuration Protocol Specification . . . . . . . . . . . 30

3.9 Configuration Protocol Diagram . . . . . . . . . . . . . . 31

3.10 ICANHAZ Message Definition . . . . . . . . . . . . . . . . 32

3.11 KVSYNC Message Definition . . . . . . . . . . . . . . . . 32

3.12 KTHXBAI Message Definition . . . . . . . . . . . . . 33

3.13 KVPUB Message Definition . . . . . . . . . . . . . . 33

3.14 HUGZ Message Definition . . . . . . . . . . . . . . . . 33

3.15 Data Flow Diagram . . . . . . . . . . . . . . . . . . . . . . 34

3.16 Data Flow Specification . . . . . . . . . . . . 35

3.17 DATA Message Definition . . . . . . . . . . . . 37

3.18 Branch Recovery Protocol . . . . . . . . . . . . . . . . . 37

3.19 Branch Recovery Protocol Diagram . . . . . . . . . . . . 38

3.20 Root Recovery Protocol _. . . . . . . . . . . . . . . 38

3.21 Root Recovery Protocol Diagram . . . . . . . . . . . . . . . 39

4.1 Recursive 25th Fibonacci PHP Script . . . . . . . . . . . . . . 42

4.2 Transparency - 25th Fibonacci . . . . . . . . . . . . . . . 44 
4.3 Transparency - 5MB Download . . . . . . . . . . . . . 45

4.4 Scalability - Steady-State Network Bytes . . . . . . . . . . . . . . 47

4.5 Scalability - Steady-State Network Packets . . . . . . . . . . . . . 48

4.6 Scalability - Average Network Utilization . . . . . . . . . . . . . 49

4.7 Scalability - Average Network Utilization Per Node . . . . . . . . 50 


\section{CHAPTER 1}

\section{Introduction}

As the Internet has become more pervasive in today's business economy, there has been a natural trend of distributing large, complex systems across multiple components locally and throughout the world. These systems are not always homogeneous with respect to hardware architecture or even operating system, and development of these systems can prove to be quite difficult even with the best tools available. In order to effectively build these systems, software engineers must be able to test their system for performance defects as well as bottlenecks. Additionally, distributed systems must respond to changes in availability and work load on its individual nodes.

Distributed performance testing frameworks supply software practitioners and system administrators with tools to evaluate the performance of a system from both black box and white box perspectives by publishing interfaces for instrumenting, collecting, analyzing, and visualizing performance data across the distributed system and distributed applications. Distributed performance monitoring frameworks, often considered part of the testing framework, provide a black box interface into monitoring a distributed system or application and usually includes mechanisms for triggering actions based on performance events. For the purpose of this work, the term distributed performance framework is introduced to collectively refer to both distributed performance testing and distributed performance monitoring frameworks. 


\subsection{Distributed Performance Framework Criterion}

In order for practitioners and researchers alike to effectively choose a distributed performance framework, it is necessary to have a set criteria for evaluation. Presented here is an extended criterion of the general requirements presented by [30] for grid systems. Data Delivery Models and Security have been taken directly from their work. Scalability has been modified to only consider good performance as its goal while Low Intrusiveness has been turned into Transparency. Extensibility has been removed from the list, and Completeness and Validity have been added. This work provides an alternate definition for Portability.

\subsubsection{Data Delivery Models}

Monitoring information includes fairly static (e.g., software and hardware configuration of a given node) and dynamic events (e.g., current processor load, memory), which suggests the use of different measurement policies (e.g., periodic or on demand). In addition, consumer patterns may vary from sparse interactions to long lived subscriptions for receiving a constant stream of events. In this regard, the monitoring system must support both pull and push data delivery models. [30]

\subsubsection{Security}

Certain scenarios may require a monitoring service to support security services

such as access control, single or mutual authentication of parties, and secure transport of monitoring information. [30] 


\subsubsection{Scalability}

Monitoring systems have to cope efficiently with a growing number of resources, events and users. This scalability can be achieved as a result of good performance which guarantees that a monitoring system will achieve the needed throughput within an acceptable response time in a variety of load scenarios. [30]

\subsubsection{Transparency}

Transparency refers to the lack of impact a distributed performance framework makes on the system being monitored. As [30] states, it is "typically measured as a function of host (processor, memory, I/O) and network load (bandwidth) generated by the collection, processing and distribution of events." If a framework lacks transparency it will fail to allow the underlying distributed system to perform well and will produce inaccurate performance measurements, thereby reducing its Scalability and destroying its Validity.

\subsubsection{Completeness}

The Completeness of a distributed performance framework refers to the exhaustiveness to which it gathers performance metrics. At a minimum, a framework must provide interfaces for measuring and aggregating performance data about a system's processor, memory, disk, and network usage. Several distributed performance frameworks provide further detailed performance metrics about the given distributed system being monitored, but this is usually at the cost of Portability. 


\subsubsection{Validity}

A distributed performance framework is only as good as the data is produces; if the sensors or gathering techniques are inaccurate, then the data is useless at best, misleading at worst. Validity of a framework is achieved when the authors of a framework provide formal verification of its accuracy.

\subsubsection{Portability}

A framework's ability to run on a completely heterogeneous distributed system without special considerations by the practitioner is what this work defines as Portability. More specifically, a portable framework has a unified API regardless of the system architecture, does not restrict itself to applications written in specific programming languages, and does not require practitioners to manually instrument their application code. This black box characteristic is vital for a viable distributed performance framework's effectiveness as it allows practitioners to focus on the performance data and not on a myriad of APIs for various architectures or languages.

\section{$1.2 d C A M P$}

The Distributed Common API for Measuring Performance $(d C A M P)$ is a distributed performance framework built on top of Mark Gabel and Michael Haungs' 2007 research on CAMP: a common API for measuring performance [4]. The fundamental functionality of $C A M P$ is providing an accurate and "consistent method for retrieving system performance data from multiple platforms."

$d C A M P$ takes advantage of this functionality and the authors' work done in validating $C A M P$ 's accuracy and adds the core feature sets listed below. As 
shown in the analysis work presented in Chapter 4, dCAMP adds these features while still maintaining minimal impact on the systems, processes, and networks being monitored.

The key contributions of $d C A M P$ are:

- a stateful performance API,

- distributed performance data aggregation,

- performance filters and triggers, and

- simplistic fault tolerance.

\subsubsection{Terminology}

Knowing the following terminology will make it easier to understand and discuss the $d C A M P$ project, its main goals, its usage, its components, and its inner workings.

\section{Distributed Performance Testing Framework (DPTF),}

\section{Distributed Performance Monitoring Framework (DPMF),}

Distributed Performance Framework (DPF): An DPTF or DPMF (collectively termed DPF) is a framework which allows its users to evaluate the performance of a system from both black box and white box perspectives by publishing interfaces for instrumenting, collecting, analyzing, and visualizing performance data across the distributed system and distributed applications. Typically, the framework provides a black box interface into monitoring a distributed system or application and includes mechanisms for triggering actions based on performance events. The $d C A M P$ project is designed to be a DPF.

\section{Performance Metric,}


Performance Counter: Performance metrics are any data about a given node relating to its throughput, capacity, utilization, or latency. In $d C A M P$, these are grouped into four different sets of performance metrics - global, network, disk, and per-process - and a fifth set of inquiry metrics. They are described fully in section 2.5 .

Metric Aggregation: Metric aggregation is the process of combining metrics from multiple nodes into a single metric. Performance metrics, while useful at an individual system granularity, can be rather limited in value for a DPF where the goal is measurement of the distributed system as a whole. Metric aggregation provides a coarser granularity for the performance metrics, calculating a sum, average, percent, or any other mathematically relevant operation across multiple nodes in the system.

Metric Calculation: Metric calculation is the process of combining identical metrics from multiple timestamps into a single metric. Various equations and inputs are used to do this calculation, chosen depending on the type of metric and desired representation; these equations are listed in Table 3.1.

\section{Filter,}

\section{Throttle,}

Threshold: Filtering (or throttling or thresholding) provides a mechanism for reducing the amount of data sent between nodes of the system. Filtering allows a user to specify when or at what point to report metrics from one level to its parent. For example, a filter might be set to only report average CPU utilization that is over seventy-five percent.

\section{dCAMP Node,}

dCAMP Process: A single, independently running instance of $d C A M P$ in the distributed system is called a $d C A M P$ node or process. More than one 
node may exist on a single computer. A node consists of the Node role and zero or more other $d C A M P$ roles.

$\boldsymbol{d} \boldsymbol{C A} \boldsymbol{M P}$ Service: Services are a way of logically grouping functions within the $d C A M P$ system, from performance metric sampling to $d C A M P$ system management. A description of all the $d C A M P$ services can be found in section 2.3. Each service is implemented in $d C A M P$ as an independent thread.

$\boldsymbol{d} \boldsymbol{C A} \boldsymbol{M P}$ Role: Roles in the $d C A M P$ system are groupings of one or more $d C A M P$ services. There does not exist a one-to-one relationship between roles and services; the $d C A M P$ role-to-service mapping can be seen in Table 2.1.

$\boldsymbol{d} \boldsymbol{C A} \boldsymbol{M P}$ Hierarchy: The $d C A M P$ system is organized in a hierarchical pattern with respect to data movement and system control functionality. The hierarchy can be thought of as a tree structure, with leaf nodes being at the top of the hierarchy and a single root node at the bottom. Metric data moves down the hierarchy from leaves to the root; configuration data and control commands move up from the root to the leaves.

$\boldsymbol{d C A} \boldsymbol{M P}$ Level: Levels are a way of organizing the $d C A M P$ hierarchy horizontally. Levels are defined by their distance from the root node. For example, level one is one node away from the root node, or said another way, the first level is directly "connected" to the root node. The second level is two nodes away from the root node, or any node in the second level is connected to the root node by another node (in the first level). This necessarily means the root is in level zero (all by itself).

Parent Node: Nodes are called parent nodes if there exists at least one node connected to it from a level of higher ordinal value. For example, a node in 
level one with at least one node connected to it from level two is considered a parent node. The root node is inherently a parent node.

Child Node: A node is called a child node if it is connected to another node in a level of lower ordinal value. For example, a node in level one is connected to the root node (in level zero), so it is called a child node. The root node is the only node in the $d C A M P$ system which is not a child node.

$\boldsymbol{d C A} \boldsymbol{M P}$ Configuration: The $d C A M P$ configuration specifies everything about the system, including hierarchy levels, metrics, sampling periods, reporting periods, filtering, communication details, etc. The configuration is set at the root node and then distributed to the rest of the $d C A M P$ system. Configuration details can be found in section 2.6.

\section{ZeroMQ,}

zmq,

ØMQ: ZeroMQ is a message queuing framework which allows a developer to build distributed systems by focusing on the data and implementing simple design patterns.

ZeroMQ Address: A $Ø \mathrm{MQ}$ address is the combination of network host identifier (i.e. an IP Address or resolvable name) and Internet socket port number.

ZeroMQ Endpoint: An endpoint is the combination of any ZeroMQ transport (pgm, inproc, ipc, or tcp) and a ØMQ address.

\section{Metric Collection,}

Metric Sampling: Metric collection or sampling is the process of measuring metrics on a given node.

Metric Reporting: Metric reporting is the process of sending sampled metrics to a parent node. 


\title{
CHAPTER 2
}

\author{
Design
}

$d C A M P$ is designed to be simple and only add complexity where needed. This allows for quick and easy, large scale testing, for example. Additionally, in order to be transparent, minimizing network traffic was an important concern.

To this end, $d C A M P$ configuration and management protocols are designed to be human-readable and verbose; this makes them easy to debug and modify as needed. The data protocol, while also human-readable in its current form, is terse with respect to the number of required messages and can be easily modified to use a more compact encoding scheme.

\subsection{Architecture}

$d C A M P$ is designed as a semi-centralized, hierarchical peer-to-peer system utilizing the UNIX Pipes and Filter architectural pattern [6] in which leaf (Metric) nodes of the hierarchy collect data, filter out extraneous data, and send it up the pipe to a parent (Collector) node which subsequently filters out more data and sends it up to another parent (Collector or Root) node. This architecture is efficient in that unwanted data is discarded earlier in the data path, reducing transport and processing costs. 


\subsection{Requirements}

As with any software engineering project, it is vital to have clearly stated requirements. $d C A M P$ is no different. Below are the list of functional and non-functional requirements which guide its design and implementation.

\subsubsection{Functional}

1. Configuration and Management

- An interface to instantiate and administer the system MUST be provided.

- Topology coordination MUST be handled automatically.

- An interface for configuring metric collections MUST be provided.

2. Metric Collection

- An API for stateful, aggregate metrics on top of CAMP must be provided.

- Filters and thresholds SHOULD be configurable at any level in the collection topology.

- Aggregation of metrics across nodes MUST be supported.

- Performance data SHOULD be written to log files on each node.

3. Fault Tolerance

- The topology MUST sustain brief network disconnectivity of any node.

- The topology MUST handle entrance/exit of any node(s) in the system.

- Metric nodes MUST be allowed to enter/exit topology at any time. 
- Root/Collector nodes (i.e. parents) MUST failover in case of extended disconnectivity.

- Loss of previously collected data SHOULD be minimized during failover.

- Management node MAY be allowed to enter/exit topology at any time.

\subsubsection{Non-Functional}

1. Transparency: $d C A M P$ SHOULD introduce negligible performance impact on Metric nodes.

2. Accuracy: dCAMP MUST accurately report performance of Metric nodes (individual and aggregated).

3. Scalability: $d C A M P$ SHOULD maintain its transparency and accuracy as it scales (i.e. the number of Metric nodes increases).

\section{$2.3 \quad d C A M P$ Roles and Services}

The $d C A M P$ distributed system is comprised of one or more nodes, each executing a role. The role is essentially a named grouping of a specific, known set of functionality or service. Roles have little to no actual run-time logic but simply act as containers for the services; services manage ZeroMQ sockets, communicating with other services/nodes, and do the real work of $d C A M P$.

\subsubsection{Services}

Each $d C A M P$ service has a specific purpose, but its scope can vary depending on the node's level in the $d C A M P$ topology. 
For example, the Configuration service has a specific purpose of replicating the $d C A M P$ configuration from the Root to every node in the system via three distinct scopes: root, branch, and leaf. As part of the Root node, the Configuration service acts as a master copy of the configuration and publishes new values as needed. As part of a Collector node, the Configuration service stores every update from the Root (for possible use if the Root dies) but no other changes are allowed to be written. Lastly as part of a Metric node, only configuration updates relevant to the node are stored by the Configuration service.

- Node - rudimentary $d C A M P$ functionality; handles topology communication, heartbeat monitoring, and failure recovery.

- Sensor-local performance metric gathering; essentially the $d C A M P$ layer on top of the OS and hardware performance APIs (accessed via CAMP).

- Filter-performance metric filtering; provides throttling and thresholding of metrics.

- Aggregation-performance metric aggregation; provides collection of and calculation on metrics from multiple Sensor and/or Aggregation services.

- Management-primary entry-point for end-user control of $d C A M P$ distributed system; this is the $d C A M P$ instrument panel, providing basic administration functions (e.g. start, stop, etc.).

- Configuration-complete or partial configuration replication; provides topology and configuration distribution.

\subsubsection{Roles}

The Base role must be running on each node for it to be part of the $d C A M P$ distributed system. In this document, a "Base node" is defined as a $d C A M P$ node which has not yet been configured, i.e. it has not joined a running $d C A M P$ 


\begin{tabular}{ll}
\hline Role & Service(s) \\
\hline Root & Management, Aggregation, Filter, Configuration (Full) \\
Collector & Aggregation, Filter, Configuration (Full) \\
Metric & Sensor, Filter, Configuration (Partial) \\
Base & Node
\end{tabular}

\section{Table 2.1 - Role to Service Mappings}

system. All other roles are launched from within the Base role; see Section 3.1.2 for more details.

The Metric role runs on the nodes from which performance metrics should be collected. The Collector role acts as an aggregation point in the system, combining performance data from multiple Metric (and Collector) nodes and providing additional aggregated performance metrics.

There is only one Root role active in the system; it acts as the master copy of the $d C A M P$ configuration and sole user-interface point. The Root role is not strictly attached to any given node in the system. Rather, the Root role may dynamically move to any first-level Collector node if the current Root node fails.

Depending on the use case and desired system performance, an administrator may choose to split roles across multiple nodes or collapse them onto a single node. For example, a single node may act as Metric, Collector, and Root for smaller systems while larger systems would employ dedicated Collector nodes. The $d C A M P$ Configuration syntax easily provides this flexibility to the system administrator.

Table 2.1 lists the roles which can be executed by a $d C A M P$ node and the services which they implement. 


\subsection{Fault Tolerance}

In order to maintain a healthy distributed topology, $d C A M P$ quickly and efficiently recovers from node failures using simple fault tolerance rules. These rules define how and when nodes are considered to have failed as well as the specific steps for recovering and/or rebuilding the distributed topology.

\subsubsection{Heartbeating (Detecting Disconnections)}

$d C A M P$ detects node failures or disconnections via a lack of messages, e.g. missed X consecutive messages or no messages received after D seconds. All messages act as heartbeats, not just the special HUGZ messages. By designing the protocols with this in mind, network traffic can be minimized.

The $d C A M P$ node failure detection rules are the following:

- Metric nodes MUST detect when their parent (Collector) node disconnects. (Promotion Algorithm)

- The Root node MAY detect when a Collector node disconnects. (Promotion Algorithm)

- Collector nodes MUST detect when the Root node disconnects. (Election Algorithm)

- The Root node MUST detect when a Metric node rejoins the system. (Reminder Algorithm)

Because of the nature of ZeroMQ sockets, Collector nodes should not need to know when a child (Metric) node disconnects - when the child node reenters the topology, it is simply reassigned underneath the Collector and resume its metric collection and reporting. 
Alternative approaches to Collector node failure detection are (A) use of an ephemeral time-to-live (TTL) property stored by the Configuration service and (B) Collector-to-Collector heartbeating. (B) introduces additional network traffic and cannot scale with sufficiently large topologies. Furthermore, the same essential functionality is present in (A) as the TTL would propagate to all Collector nodes via the Configuration service.

While approach (A) provides an additional detection mechanism for Collector node disconnections (namely the Root would detect Collector failures as the TTL expires), it is simpler and arguably no less resilient to solely detect failures from the child node's perspective.

\subsubsection{Reminder Algorithm (Metric Node Recovery)}

Metric nodes can leave and enter the $d C A M P$ system at any time. When they rejoin, they are placed back into the same location within the topology so as to maintain as much consistency within the performance data as possible.

The crux of this algorithm is the group definitions within the $d C A M P$ configuration: nodes are always defined to be within a group, and the groups define the network topology. Essentially, this algorithm is incorporated into the Topology protocol; no additional work is necessary.

1. Metric node rejoins the system with POLO response to Root node's MARCO message.

2. Root node detects Metric node is already part of $d C A M P$ system.

3. Root node (re)sends ASSIGN message to Metric node.

Collector nodes will not be overloaded by this algorithm since Metric nodes are statically defined in groups via the $d C A M P$ Configuration. As Metric nodes 
disconnect and reconnect, the Collector node virtually always has the same child nodes beneath it.

\subsubsection{Detection}

Detecting when a Metric node disconnects is not necessary. Rather the Root node only needs to detect when a Metric node rejoins the $d C A M P$ system, comparing the Metric node's UUID to the UUID already saved in the topology.

\subsubsection{Promotion Algorithm (Collector Node Recovery)}

As with the Reminder Algorithm, this recovery relies heavily on the Topology Management Protocol. When a Metric node, M, detects its Collector node, C, is down,

1. M sends an SOS message to the Root node, R.

2. If $\mathrm{R}$ has received an SOS message from more than $1 / 3$ of C's group, the algorithm proceeds as per below.

When the Root node, R, detects one of the Collector nodes has disconnected,

1. R broadcasts a STOP message to all nodes within C's group and clears the groups configuration from the $d C A M P$ system.

2. R then broadcasts a MARCO message and begins rebuilding the group topology via the Topology protocol.

\subsubsection{Detection}

M will use the Configuration Replication Protocol (HUGZ message is sent when there are no configuration updates) to detect when $\mathrm{C}$ disconnects. $\mathrm{R}$ will use the 
Data Flow Protocol (DATA (type='HUGZ') message is sent when no data is scheduled to be reported) to detect when any of its Collector nodes has disconnected. That is, if $\mathrm{M}$ or $\mathrm{R}$ receives no messages from $\mathrm{C}$ within $\mathrm{D}$ seconds, $\mathrm{C}$ is considered disconnected.

\subsubsection{Election Algorithm (Root Node Recovery)}

This recovery algorithm is based on the bully algorithm presented by H. GarciaMolina in [5]. Only Collector nodes participate in the election, initiated when a Collector node, C, detects the Root node, $\mathrm{R}$, is down.

1. C sends WUTUP message to all nodes whose UUID is higher than its own, expecting a YO message in response.

2. If $\mathrm{C}$ does not receive any $\mathrm{YO}$ messages,

(a) C declares victory by sending IWIN message to all nodes, and

(b) $\mathrm{C}$ waits $\mathrm{W}$ seconds before transitioning to become the Root, allowing for another node to replace it as Root via a separate election.

3. If $\mathrm{C}$ receives a $\mathrm{YO}$ message,

(a) $\mathrm{C}$ waits for $\mathrm{W}$ seconds to receive an IWIN message from another node whose UUID is higher than its own.

(b) If no IWIN message is received, $\mathrm{C}$ resends its WUTUP message and goes through the election process again.

Additionally,

- If $\mathrm{C}$ receives a WUTUP message from a node whose UUID is lower than its own, C responds with a YO message and then starts its own election.

- If $\mathrm{C}$ receives an IWIN message from a node whose UUID is lower than its own, C immediately begins a new election. 


\subsubsection{Detection}

C will use the Configuration Replication Protocol (HUGZ message is sent when there are no configuration updates) to detect when $\mathrm{R}$ disconnects. That is, if $\mathrm{C}$ receives no message from $\mathrm{R}$ within $\mathrm{D}$ seconds, $\mathrm{R}$ is considered disconnected.

\section{$2.5 d C A M P$ Metrics}

The crux of any DPF are the performance metrics to which it provides access. This section lists the set of performance metrics designed within $d C A M P$. Metrics

marked with " $(d C A M P)$ " are extensions added by the $d C A M P$ project to the basic CAMP metrics. These provide a performance view of multiple nodes in the distributed network and are collected by the Aggregation service rather than the Sensor service.

It should be noted here: only a basic subset of these metrics are actually implemented in the current version of $d C A M P$ as a proof of concept. Please, refer to page 62 of the Future Work section for more details.

\subsubsection{Global Metrics}

Global metrics measure overall CPU, process, thread, and memory usage of the system.

- Node CPU usage

- Node free physical memory

- Aggregate average CPU usage $(d C A M P)$

- Aggregate free physical memory $(d C A M P)$ 


\subsubsection{Network I/O Metrics}

Network metrics measure utilization of a given network interface on the system.

- Total bytes sent on the given interface

- Total packets sent on the given interface

- Total bytes received on the given interface

- Total packets received on the given interface

- Aggregate bytes sent $(d C A M P)$

- Aggregate packets sent $(d C A M P)$

- Aggregate bytes received $(d C A M P)$

- Aggregate packets received $(d C A M P)$

\subsubsection{Disk I/O Metrics}

Disk I/O metrics measure throughput of a given disk or partition on the system.

- Number of read operations on the given disk

- Number of write operations on the given disk

- Number of read operations on the given partition

- Number of write operations on the given partition

- Aggregate number of read operations $(d C A M P)$

- Aggregate number of write operations $(d C A M P)$

\subsubsection{Per-process Metrics}

Per-process metrics measure CPU, memory, and thread usage for a single process on the system.

- Number of major and minor page faults 
- Process CPU utilization

- Process user mode CPU utilization

- Process privileged mode CPU utilization

- Size of the process' working set in KB

- Size of the used virtual address space in KB

- Number of threads contained in the process

\subsubsection{Inquiry Metrics}

Inquiry metrics provide a mechanism for enumerating various properties of the system.

- Enumeration of the available disk partitions

- Enumeration of the available physical disks

- Enumeration of the valid inputs to the network functions

- Number of CPUs in the system

- "process identifier" for the given PID

- "process identifier" for each running process launched from an executable of the given name

\subsection{Configuration}

A main feature of $d C A M P$ is the configuration language which gives a system administrator a concise, powerful tool for defining its performance monitoring behaviour. Two primary sets of parameters are needed in this configuration: the set of nodes to include in $d C A M P$ and the set of metrics those nodes will collect.

While it would be possible for $d C A M P$ to be designed such that branches of the distributed hierarchy are automatically formed based on dynamic inputs 
(e.g. node locality, performance metric configuration, balance of network vs $\mathrm{CPU} /$ memory impact), the approach taken in $d C A M P$ gives the system administrator control over this parameter.

Specifically, nodes are defined in groups within the $d C A M P$ configuration, and each group is defined to collect one or more distinct performance metrics. This gives the system administrator the freedom to collect varying metrics from each branch of the topology and/or collect metrics at varying sample periods.

\subsubsection{Node Specification}

Nodes may be specified individually (as ZeroMQ addresses) or as groups (IP subnets). Additionally, nodes may be included or excluded based on host name or IP Address matching. Name matching does a case-insensitive comparison of the node's host name; left, right, or whole name matching can be specified. Address matching checks that the node's IP Address falls within a given subnet (i.e. IP Address and mask length).

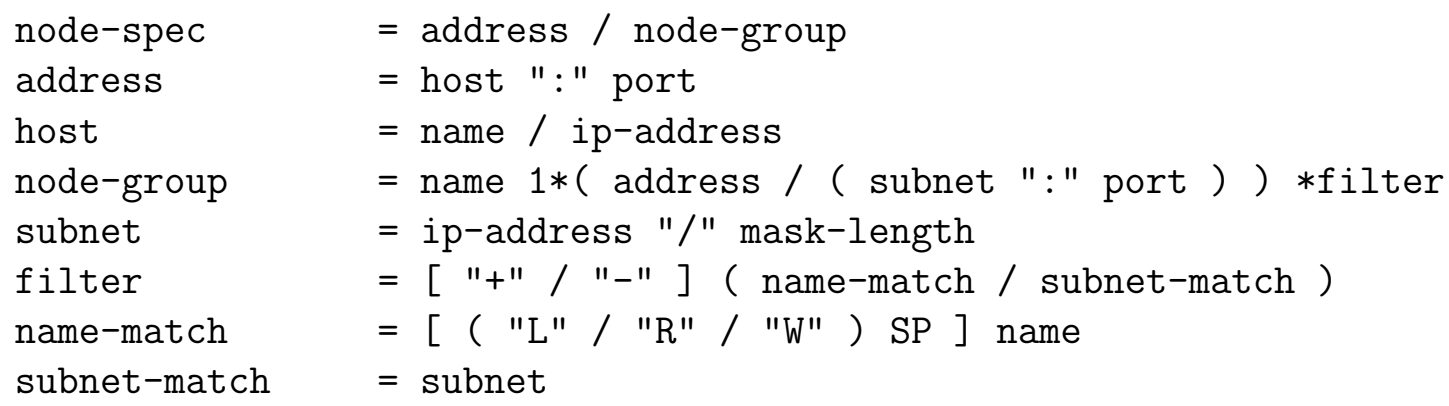

Figure 2.1 - Configuration File - Node Specification 


\subsubsection{Sample Specification}

Performance metric samples are specified as:

1. the node(s) on which to sample the data,

2. the rate at which data should be sampled,

3. the threshold past which data should be reported, and lastly

4. the actual performance metric to be sampled.

The report threshold can be specified as "hold and report every N seconds" or "report when the metric value is greater/less than X". When "hold" is specified (via an *), all metric values sampled during the time limit are sent. Otherwise, the $<$ or $>$ character indicates the metric should only be reported when its calculated value is less-than or greater-than the given threshold value.

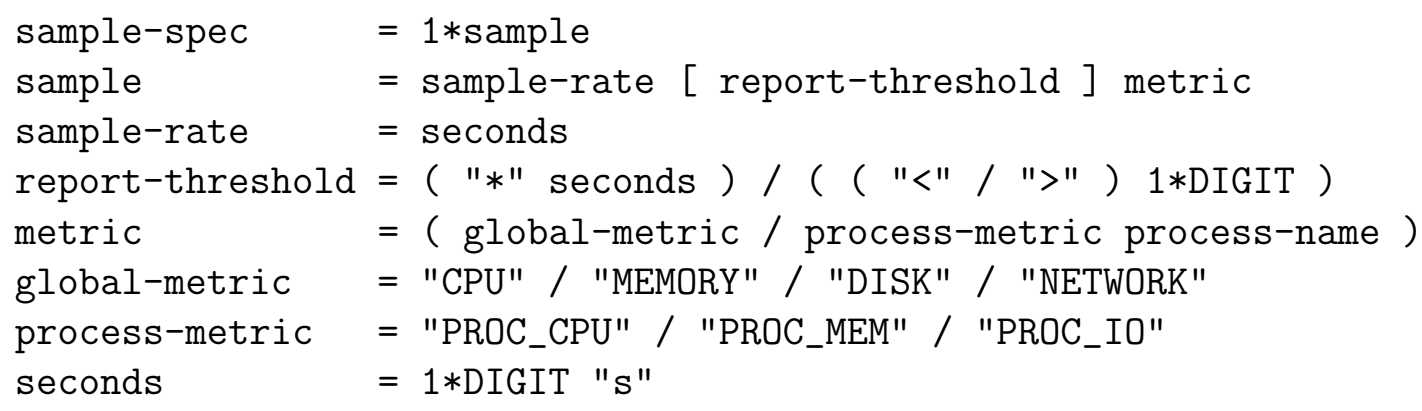

Figure 2.2 - Configuration File - Sample Specification: Only a basic subset of metrics are implemented in the current version of $d C A M P$ as a proof of concept. Please, refer to page 62 of the Future Work section for more details.

\subsubsection{Accumulative Time-Based Filtering Pitfall}

Filtering can be thought of being done in one of two ways: accumulatively or discretely. Accumulative means only one final value is reported for each time range (e.g. collect every second but report every minute, so sixty samples are 
combined into a single value and then sent). Discrete means each constituent value is sent for each time range, but they are "held" until the time limit is reached.

However, accumulation is not valuable for monotonically increasing valuesit is the same as just sampling at the slower frequency. Accumulation is only valuable for non-monotonically increasing values, but in that case, one should find the raw, monotonically increasing values from which it is calculated and collect that metric instead. 


\title{
CHAPTER 3
}

\author{
Implementation
}

\section{$3.1 \quad d C A M P$ Operation}

\subsubsection{Sequence of $d C A M P$ Operation}

The following steps describe how the $d C A M P$ system is turned on. The Base nodes (other than the node assigned to be the Root) can be started at any time by using the $d C A M P$ CLI, before or after the Root node is initialized. It is expected these Base nodes are managed by a watchdog utility that automatically restarts the node if it exits for any reason; a sample watchdog utility is shown in Figure 3.1 .

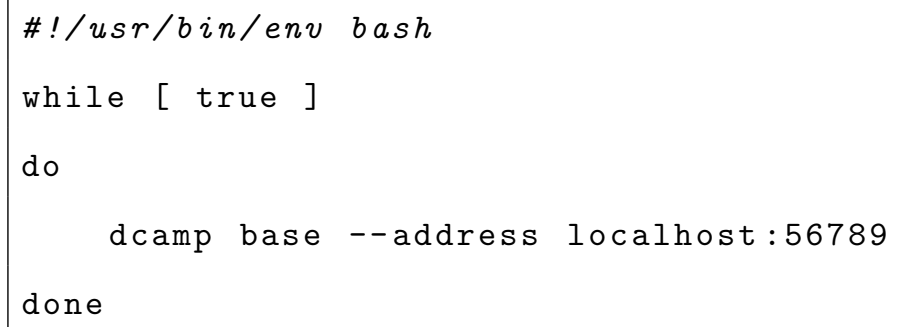

\section{Figure 3.1 - Sample Watchdog Script}

1. User promotes a Root node via the $d C A M P$ CLI, specifying a configuration file and a Base node's address.

2. Root node connects to each Base node and begins the discover Topology Protocol. 
3. Base nodes join the $d C A M P$ system at any time, being assigned as Collector or Metric nodes in the topology.

4. $d C A M P$ runs in a steady state, nodes entering or exiting the system at any time.

- Performance counters are sampled, filtered, reported, and logged by the Metric nodes at regular intervals according to the $d C A M P$ Configuration.

- Performance counters received from child nodes are aggregated, filtered, reported, and logged by Collector nodes at regular intervals according to the $d C A M P$ Configuration.

- Performance counters received from child nodes are aggregated and logged by Root node for later processing (e.g. graphing metrics during a test scenario or correlating statistics with a distributed event $\log$ ).

5. User stops $d C A M P$ by using the $d C A M P$ CLI command.

6. Root node begins the stop Topology Protocol.

7. Collector and Metric nodes exit the topology and revert to Base nodes.

8. Root node exits, reverting to Base node.

\subsubsection{Threading Model}

As mentioned above as the first and third steps of $d C A M P$ operation, a Base node can transform into one of the three active $d C A M P$ roles: Root, Collector, or Metric. This transformation is actually the Base role (via the Node service) launching and managing another role internally. This interaction is depicted in Figure 3.2. 


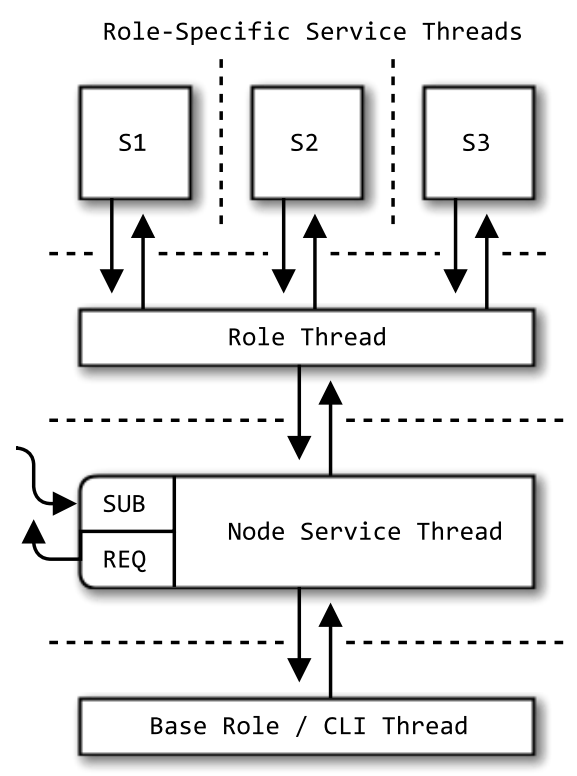

Figure 3.2 - Node, Role, Services Threading Model Diagram: Thread boundaries are represented by dashed lines. Except for the Node service's SUB and REQ sockets, all arrows represent PAIR socket communication.

When a Base node is running, only the bottom two threads (the Base role and the Node service) are active. Once it receives an assignment from the discover Topology Protocol or the $d C A M P$ CLI, the Node service launches an appropriate role thread which, in turn, launches one or more role-specific service threads.

All communication between the roles and services occurs across PAIR control sockets. There are also various service-to-service communications which occur via inproc transport sockets (e.g. the internal Data Flow Protocol) and shared memory data structures (e.g. the Configuration service).

Also mentioned in section 3.1.1 as the last two steps, each role exits and, by doing so, reverts itself back to a Base node. This is handled just like before, with the Node service receiving a STOP message via the stop Topology Protocol and then notifying the internally running role to shut down. The role thread then 
notifies its service threads, waits for them to finish, then exits.

\subsection{ZeroMQ Protocols}

ZeroMQ is a fantastic message queuing framework that essentially provides more intelligent sockets as building blocks for distributed systems. ZeroMQ handles the intricacies of sending messages between two endpoints and lets the application handle the rest of the logic. The protocols described in this section do not come from ZeroMQ, rather they are built using ZeroMQ sockets and message patterns.

For a quick background on ZeroMQ socket types and message patterns, please see Appendix A.

\subsubsection{Topology Protocols}

The $d C A M P$ distributed topology is dynamically established as the Root node sends out its discovery message and receives join messages from Base nodes. When a Base node responds to the Root, the Base node is given its assignment.

To reduce network traffic and load on the Root, Base nodes are designed to ignore MARCO messages from nodes whose UUID matches a previous successful topology discovery handshake. The Root node uses this to its advantage when attempting to stop nodes: the same MARCO / POLO pattern is used, but the Root node uses a different UUID in the MARCO message and a responds with a STOP message instead of an assignment.

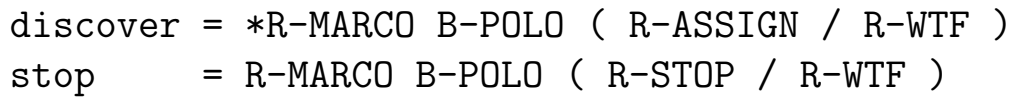

Figure 3.3 - Topology Protocols: R- represents the Root node sending a message and B- represents a Base node sending a message. 


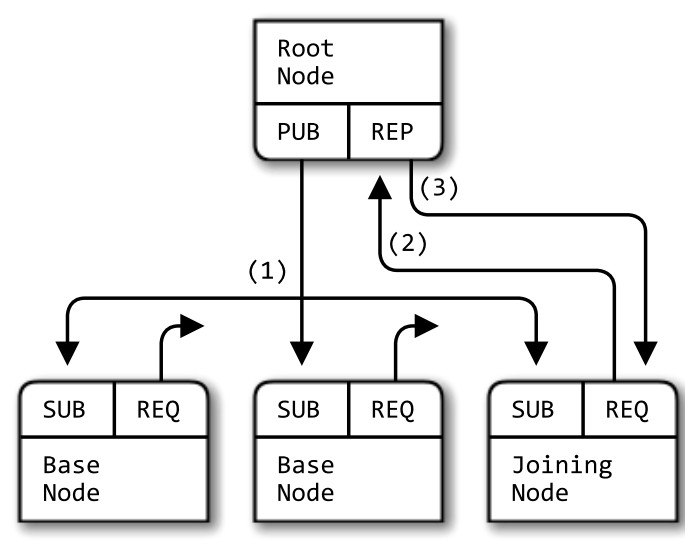

Figure 3.4 - Topology Protocol Diagram: (1) Root sends MARCO at regular intervals, (2) Base sends POLO request, (3) Root replies with ASSIGN or STOP

\subsubsection{Message Definitions}

TOPO is a generic topology message consisting of four frames. This message type is designed to be sent across a PUB/SUB connection, from which subscribers filter incoming messages using the first frame. This design proves useful for the Recovery Protocols.

The MARCO message is simply shorthand for TOPO (key="/MARCO").

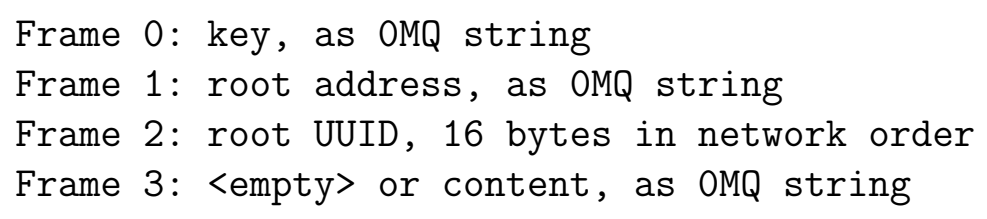

Figure 3.5 - TOPO Message Definition

CONTROL is a generic control message consisting of four frames and designed to be sent across a REQ/REP connection. The POLO, ASSIGN, and STOP messages are shorthand for CONTROL (command="POLO"), CONTROL (command="ASSIGN"), and CONTROL (command="STOP") respectively. 
In the case of ASSIGN, the third frame contains the specific topology instructions (level-one collector, leaf node, etc.) being sent to the Base node.

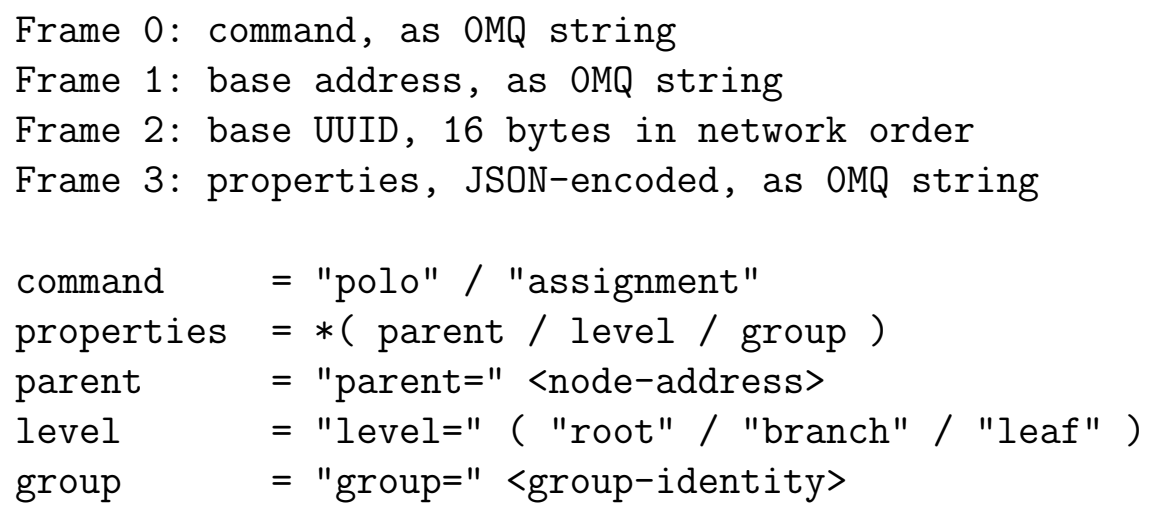

Figure 3.6 - CONTROL Message Definition

WTF is $d C A M P$ 's error message type. It has three frames (though Frame 2 may be empty) with the first designed to make error detection simple.

Frame 0: "WTF", as OMQ string

Frame 1: error code, 4 bytes in network order

Frame 2: <empty> or error message, as OMQ string

\section{Figure 3.7 - WTF Message Definition}

\subsubsection{Configuration Replication Protocol}

$d C A M P$ configuration and topology state are replicated across the system using key-value pairs, with the keys laid out in a hierarchical fashion. This lends itself nicely to PUB/SUB topic filtering.

For example, because a Metric node only needs the configuration values for its particular group, the node subscribes only to the "/CONFIG/<group-name>/" topic. Any KVPUB whose key does not start with this string is then discarded.

In practice, Metric nodes need more than just their group-specific configuration, but the general principle holds true: nodes only receive the configuration 
data they require and nothing more. In the case of first-level Collector nodes, they receive all updates since they are fail-over candidates for the Root node.

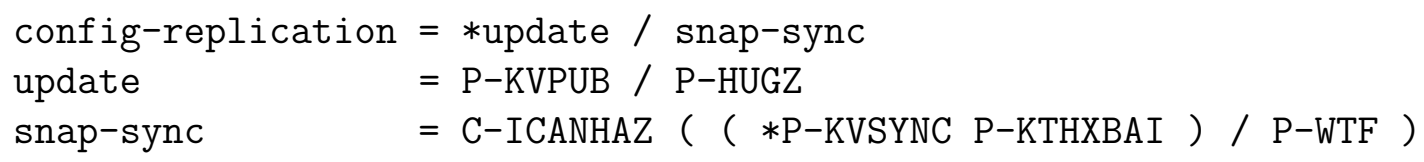

Figure 3.8 - Configuration Protocol Specification: P- represents the parent node (Root or Collector) sending a message and C- represents the child node (Collector or Metric) sending a message.

A newly assigned first-level Collector node will first subscribe to new configuration updates from the Root node and then send a configuration snapshot request to the Root node. A newly assigned Metric (or non-first-level Collector) node will first subscribe to new configuration updates from its parent Collector node, and then send its parent Collector node a filtered configuration snapshot request. Once its snapshot has been successfully received, a node will process any pending configuration updates and then, in the case of a Collector node, respond to child node snapshot requests.

The $d C A M P$ configuration replication algorithm adheres to the Clustered Hashmap Protocol[12] with a few minor (and one major) modifications:

1. only the Root node MUST write updates to the configuration,

2. the full configuration table MUST be replicated across all first-level Collector nodes (lower-level nodes MAY filter their configuration to only store relevant data),

3. a different set of command names are used (as described below), and

4. configuration updates are distributed via the $d C A M P$ hierarchy (instead of directly from the Root node). 


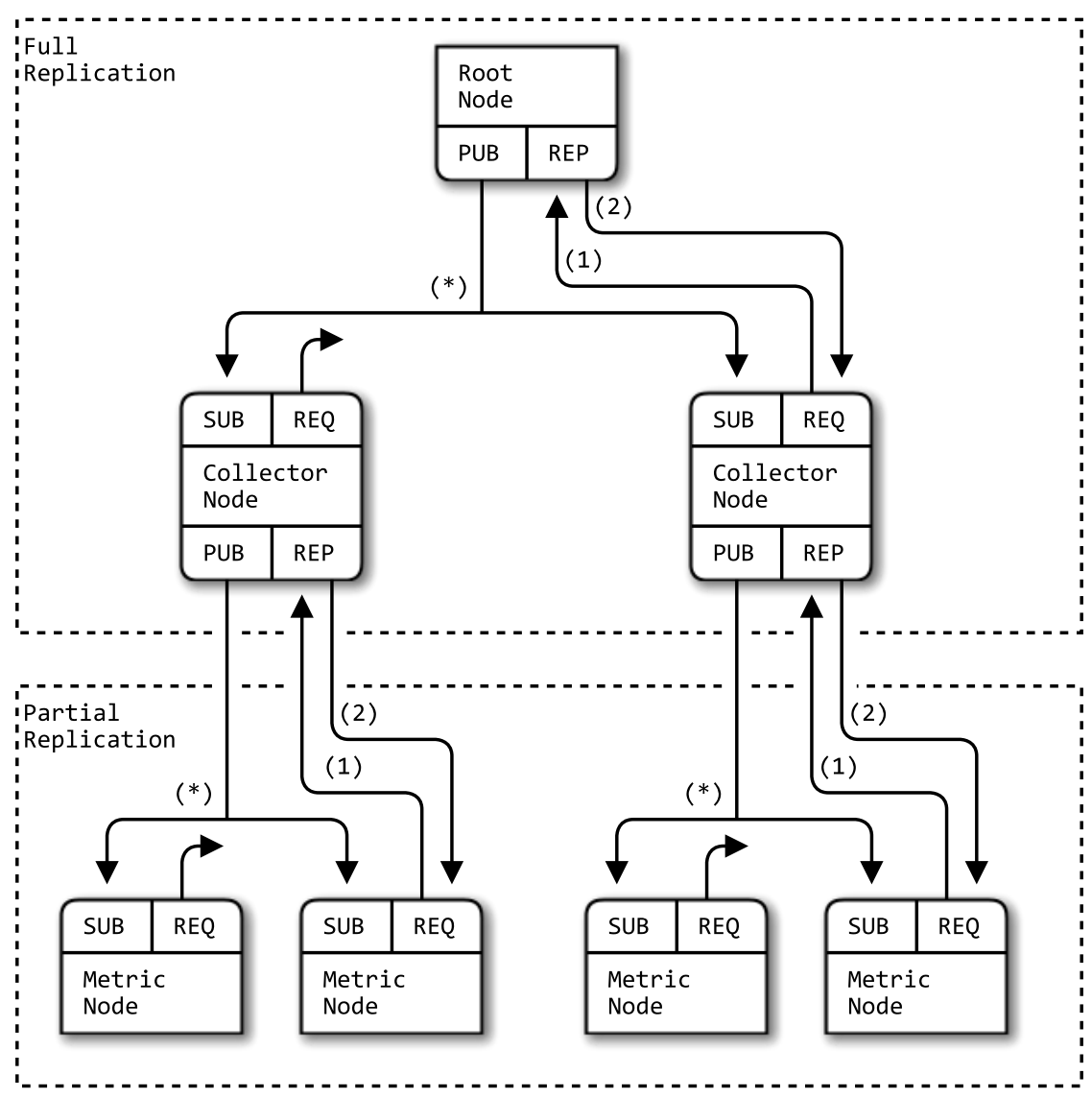

Figure 3.9 - Configuration Protocol Diagram: (*) Parent node sends KVPUB or HUGZ at any time, (1) child node sends ICANHAZ request, (2) parent node replies with zero or more KVSYNC messages followed by exactly one KTHXBAI message.

\subsubsection{Message Definitions}

These messages come from the CHP protocol. Additionally, a WTF error message may be sent by the parent in case of error. It should be noted, each of the following messages is really the same five-frame format with varying keys and semantics.

As shown in Figure 3.8, the ICANHAZ, KVSYNC, and KTHXBAI messages are sent across a REQ/REP connection type. KVPUB (as the name would imply) along 
with the HUGZ heartbeat message are designed for the PUB/SUB pattern.

ICANHAZ is a configuration snapshot request sent by the child node when it first starts. Multiple ICANHAZ requests can be sent for the different topics or subtrees needed by the node, and the node will not begin normal operation until all of the requested values have been received.

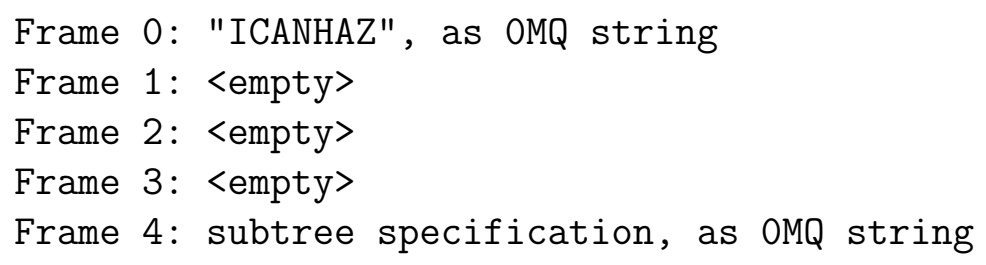

Figure 3.10 - ICANHAZ Message Definition

KVSYNC is a configuration snapshot response message. For every key-value pair within the requested subtree, a KVSYNC message is sent to the child node. Note: if no values exist for a requested subtree, a KTHXBAI message will be the only response received by the child node.

The sequence number in Frame 1 SHOULD be ignored by the recipient since no order guarantees exist for configuration snapshots requests.

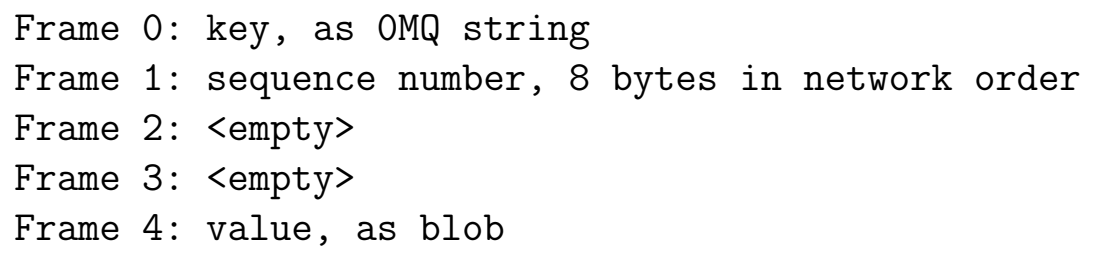

Figure 3.11 - KVSYNC Message Definition

KTHXBAI marks the end of a successful snapshot request. Frame 4 MUST contain the highest sequence number of all the values in the configuration snapshot. 


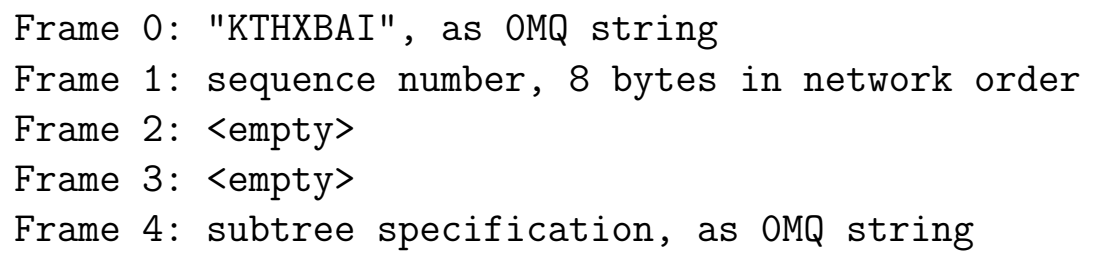

Figure 3.12 - KTHXBAI Message Definition

KVPUB is a configuration update sent from parent to child. The sequence number in Frame 1 must be monotonically increasing. When a KVPUB is received which has a sequence number lower than a previously received KVPUB, the node MUST delete its saved configuration values and request a new snapshot.

Frame 2 SHOULD contain the UUID of the node from which the value originated. In $d C A M P$, this should only be the Root node's UUID. Frame 3 MAY contain additional properties for the key-value pair, such as an ephemeral timeto-live.

Frame 0: key, as OMQ string

Frame 1: sequence number, 8 bytes in network order

Frame 2: UUID, 16 bytes in network order

Frame 3: properties, JSON-encoded, as OMQ string

Frame 4: value, as blob

Figure 3.13 - KVPUB Message Definition

HUGZ is the heartbeat message sent from parent to child when the rate of KVPUB messages being sent drops below a configured threshold. The HUGZ message is critical to maintaining topological consistency in $d C A M P$.

Frame 0: "HUGZ"

Frame 1: 00000000

Frame 2: <empty>

Frame 3: <empty>

Frame 4: <empty>

Figure 3.14 - HUGZ Message Definition 


\subsubsection{Data Flow Protocol}

There are two data flow protocols in the $d C A M P$ system: the external protocol for data flowing from one node to the next (via PUB/SUB) and the internal protocol for data flowing between components of a single node (via PUSH/PULL). Both protocols have the same specification and use the same message formats.
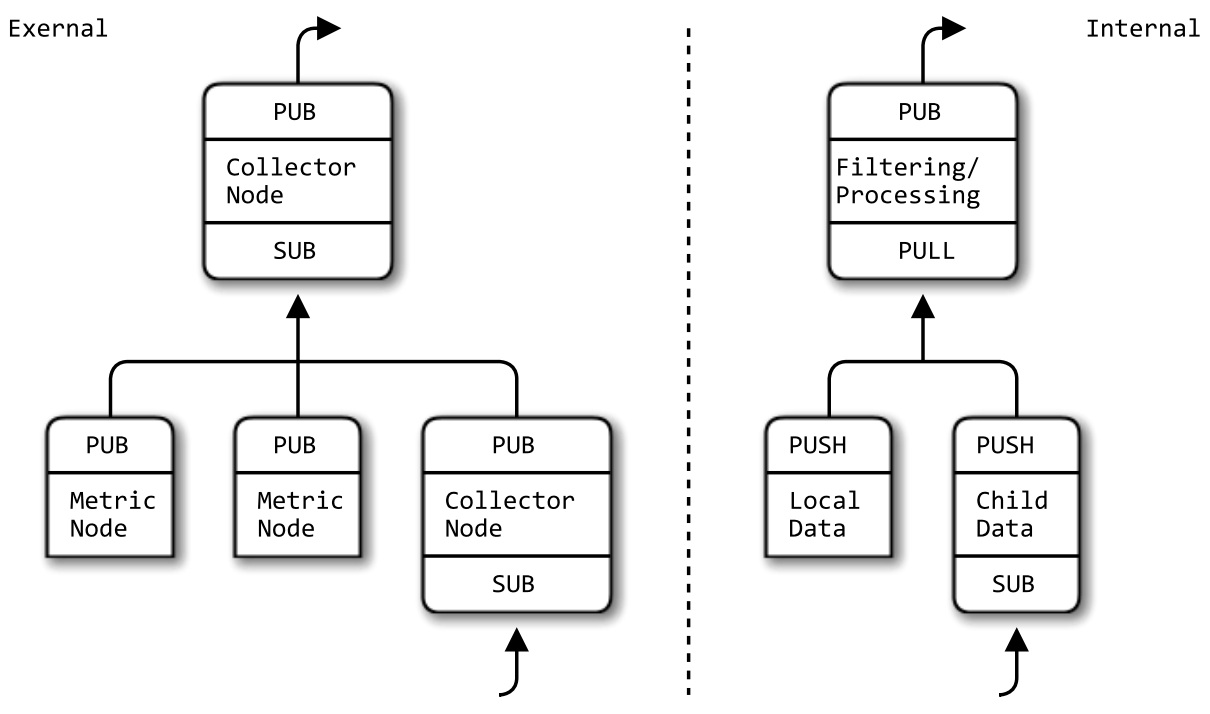

Figure 3.15 - Data Flow Diagram

The $d C A M P$ data flow protocol is very simple, comprised of a single data message type. The data flows from one node to another via PUB/SUB sockets. Internally, data flows from the upstream data producers, through a filtering/processing unit, and out to downstream data consumers via PUSH/PULL sockets.

When data rate is slower than a predefined threshold, heartbeats are sent instead to keep inter-node connections alive. 
data-flow $=*($ DATA $/$ HUGZ $)$

Figure 3.16 - Data Flow Specification: All messages are sent from child (Metric or Collector) to parent (Collector or Root).

\subsubsection{Performance Measurement}

When discussing performance measurement, it is important to understand how metrics are sampled, calculated, and presented to an end user.

Performance metrics, also called counters, are usually monotonically increasing values. That is, reading its raw, instantaneous value is virtually meaningless; to correctly read the counter it must be sampled at two different points in time and then calculated.

For example, when displaying a graph of data points for non-basic metric types, each data point is really the result of a calculation involving the metric's value at the current timestamp and that at a previous timestamp. It is possible to look at fewer data samples to first get a course-grain view (e.g. five-minute samples) of the metric before drilling in and looking at finer-grain samples (e.g. one-second samples).

Non-monotonically increasing counters do exist (e.g. disk speed, Ethernet uplink speed, etc.), but these are usually fairly static configuration values and do not need to be sampled frequently. $d C A M P$ supports these types of counters with the "basic" metric type.

Table 3.1 shows how each of the $d C A M P$ metric types are calculated. Note: unlike some other performance measurement frameworks[17], $d C A M P$ stores all metrics in their raw, uncalculated form and only presents a calculated value upon display. 


\begin{tabular}{l|l|l}
\hline Type & Contents of Single Sample & Calculation of Two Samples \\
\hline basic & raw value at timestamp & $C=V_{t_{2}}$ \\
delta & raw value at timestamp & $C=V_{t_{2}}-V_{t_{1}}$ \\
rate & raw value at timestamp & $C=\frac{V_{t_{2}}-V_{t_{1}}}{t_{2}-t_{1}}$ \\
average & raw value and base value at timestamp & $C=\frac{V_{t_{2}}-V_{t_{1}}}{B_{t_{2}}-B_{t_{1}}}$ \\
percent & raw value and base value at timestamp & $C=100 \frac{V_{t_{2}}-V_{t_{1}}}{B_{t_{2}}-B_{t_{1}}}$
\end{tabular}

Table 3.1 - Metric Types: $C$ represents the value calculated from two samples taken at $t_{1}$ and $t_{2} . V$ is the value and $B$ is the base value in the DATA message

\subsubsection{Message Definitions}

DATA is a five-frame message containing the performance metric data sampled by the Sensor service or calculated by the Aggregation service. The HUGZ message is simply shorthand for DATA (type="HUGZ").

A single data sample MUST contain: source identifier (node or aggregation), metric identifier, timestamp, and one or two values depending on the metric type.

In case of HUGZ, no other property strings are used, and Frames 3 through 5 are all empty. Frame 4 will be non-empty for average and percent types. 


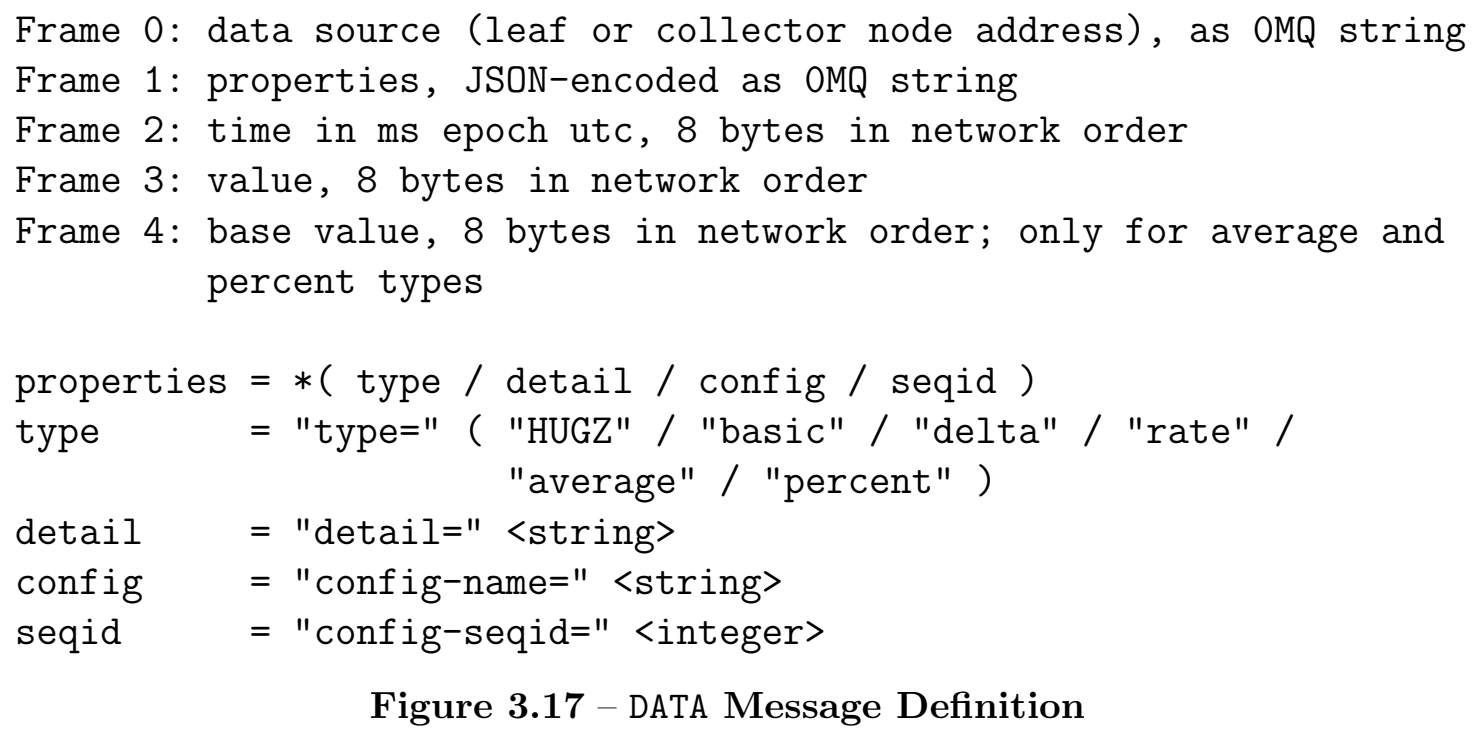

Figure 3.17 - DATA Message Definition

\subsubsection{Recovery Protocols}

The $d C A M P$ Recovery Protocols are used for the Promotion and Election algorithms and use the same base messages as the Topology Protocol, TOPO and CONTROL.

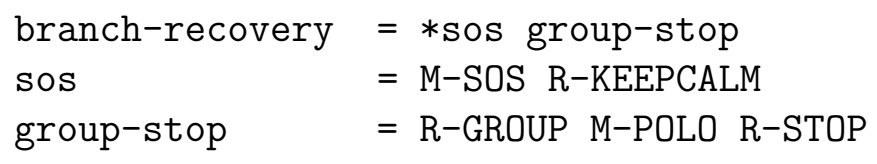

Figure 3.18 - Branch Recovery Protocol: R- represents the Root node sending a message and $\mathrm{M}$ - represents a Metric node sending a message.

The Branch Recovery Protocol is initiated by Metric nodes when they detect their Collector has died. Once the Root node has received an SOS message from at least one third of the branch's Metric nodes, the Root proceeds to shutdown the entire branch using the stop Topology Protocol. Once shut down, a new Collector is selected and the branch is rebuilt using the standard discover Topology Protocol. 


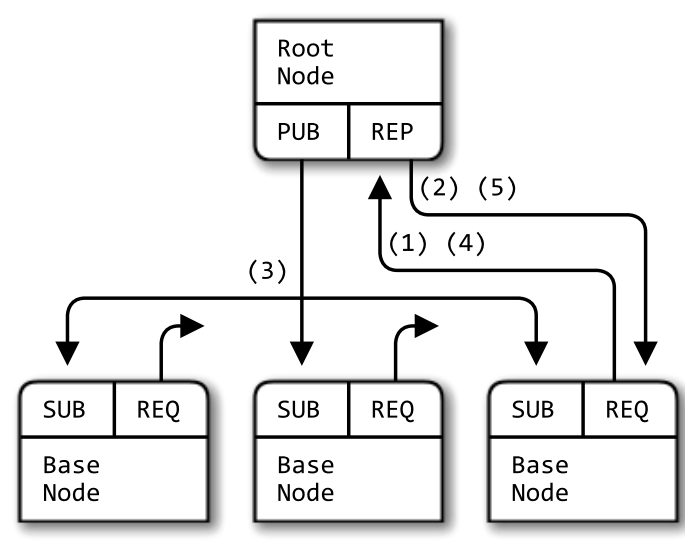

Figure 3.19 - Branch Recovery Protocol Diagram: (1) Metric nodes send SOS requests, (2) Root replies with KEEPCALM, (3) Root sends GROUP only to nodes in branch, (4) Metric nodes send POLO requests, (5) Root replies with STOP

SOS and KEEPCALM are shorthand for the CONTROL message with a command value of "sos" and "keepcalm" respectively. The POLO and STOP messages come directly from the Topology Protocol.

The GROUP message is similarly shorthand for the TOPO message with a key value of "/GROUP/<group-name>". This takes advantage of ZeroMQ's PUB/SUB filtering to only stop the faulty branch.

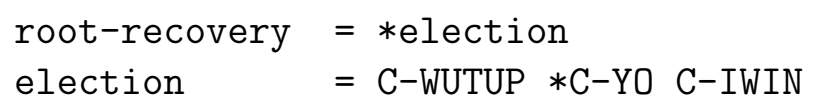

Figure 3.20 - Root Recovery Protocol: C- represents a Collector node sending a message.

As each Collector node detects the Root node has died, it attempts to start an election via the WUTUP message. Collector nodes with higher UUIDs will respond to the first Collector by sending the YO message. If no YO messages are received 
by the first Collector, the IWIN message is sent out to all Collector nodes, selfdeclaring the first Collector as the new Root.

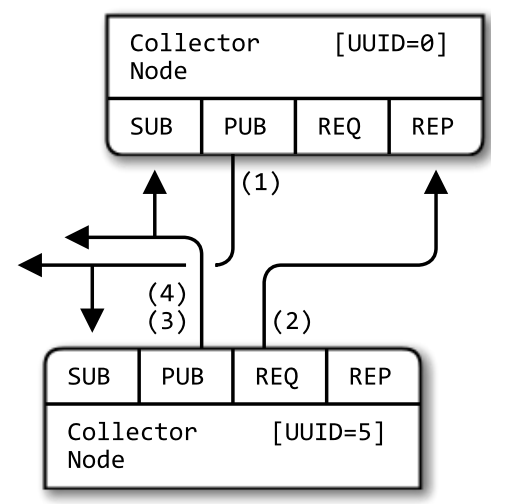

Figure 3.21 - Root Recovery Protocol Diagram: (1) WUTUP, (2) YO, (3) WUTUP, (4) IWIN

The WUTUP and IWIN messages are shorthand for TOPO (key="/RECOVERY/wutup" and TOPO (key=" /RECOVERY/iwin" respectively. The YO message is shorthand for CONTROL ( command="yo" ). 


\title{
CHAPTER 4
}

\begin{abstract}
Analysis
To verify $d C A M P$ meets both the transparency and scalability distributed performance framework criterion outlined in Chapter 1, several experiments were run on an installation of $d C A M P$ in a test environment. The goal of these experiments was two-fold: measure $d C A M P$ 's transparency in a real-world environment as well as determine the thresholds for several key configuration parameters as $d C A M P$ scales.
\end{abstract}

Any DPF can be configured in such a way that it impacts the performance of the system being monitored, for example by collecting and reporting every available global metric and per-process metrics at the fastest sampling period. Therefore, it is necessary for the system administrator to know what reasonable configuration values should be used to monitor a given distributed system.

Likewise, for $d C A M P$ to scale, it is important for the number of child nodes per parent to be limited to a reasonable number. These experiments help to define "reasonable" for various scenarios, environments, and performance monitoring requirements.

\subsection{Transparency}

To measure the impact of $d C A M P$ on a monitored process, a workload is defined and measured with and without $d C A M P$ active. The measured difference 
in performance of the monitored process is defined to be $d C A M P$ 's monitoring overhead.

\subsubsection{Workload}

Apache JMeter[15] (v2.11) is used to run load against a default-configured Apache instance on a Lenovo Thinkpad (dual 2.16GHz Centrino Duo T2600, 2GB 667MHz DDR2, SATA) running Ubuntu 13.10. The client machine, a MacBook Pro $(2.7 \mathrm{GHz}$ Core i7, $8 \mathrm{~GB} 1333 \mathrm{MHz}$ DDR3, SSD) running OSX 10.9, is directly connected to the Apache server via crossover gigabit Ethernet. When $d C A M P$ is active, a Metric node is running on the server, reporting data to a Collector node running on the client machine. The Root node is also running on the client.

Each test run includes 18 different load points, scaling the number of client threads from 2 to 2048. For every load point, the threads continuously (in this order)

1. load a static home page,

2. load a PHP page which calculates the 25th Fibonacci number (see Figure 4.1), and

3. download a 5MB file of random binary data.

The 25th Fibonacci workload is CPU-bound, and the 5MB download is IObound; the home page workload is only used to seed the client connection and is not part of the analysis measurements. After the ramp up phase of each load point (launching 10 threads per second), the test ensures all threads continue to execute simultaneously for five minutes before shutting down.

The arithmetic mean of the request latency for each step at each load point is then calculated and averaged across three distinct runs of the same test. 


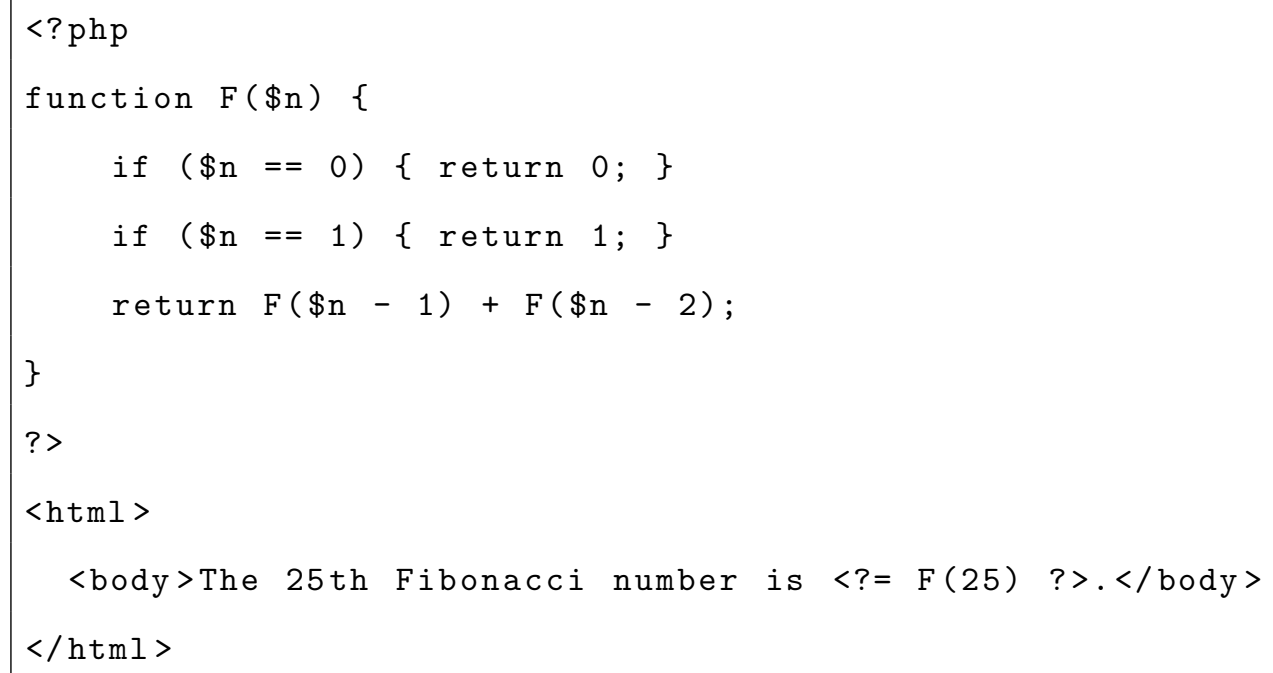

Figure 4.1 - Recursive 25th Fibonacci PHP Script: A naive approach was used in the implementation of $\mathrm{F}()$ in order to put more load on the server CPU.

\subsection{2 dCAMP Configuration}

Each $d C A M P$ configuration level monitors four global metrics and three processspecific metrics on the Apache process(es). The global metrics are CPU usage (proc), memory usage (mem), disk throughput (disk), and network throughput (net); the Apache metrics are CPU usage (apache_cpu), memory usage (apache_mem), and combined disk/network throughput (apache_io). Below are the various sample periods used for the transparency test runs.

- baseline - $d C A M P$ off

- $5 \mathbf{m}$ - all metrics every 300 seconds, heartbeats every 60 seconds

- $\mathbf{1 m}$ - all metrics every 60 seconds, heartbeats every 60 seconds

- 10s - global metrics every 300 seconds, Apache metrics every 10 seconds, heartbeats every 300 seconds

- 1s - global metrics every 300 seconds, Apache metrics every 1 second, heartbeats every 300 seconds 
No thresholds were defined for any of the above configurations. That is, Metric nodes immediately reported every sample instead of holding them for later reporting.

\subsubsection{Results}

In the CPU-bound Fibonacci test, the biggest relative increase in request latency occurs between the runs with two and four threads. This correlates to the two physical CPU cores on the system exceeding capacity. The $1 \mathrm{~m}$ config run exhibits the worst performance of all the CPU-bound tests. This shows that global metric monitoring is actually more CPU intensive than collecting per-process metrics, even for processes with many active processes.

The rate at which request latency worsens begins to level off starting at the 512 thread load point. This is also the load point at which Apache begins to return errors. As the percentage of requests resulting in errors increases, the latency of the successful requests improves slightly. This explains the trend line shift. 


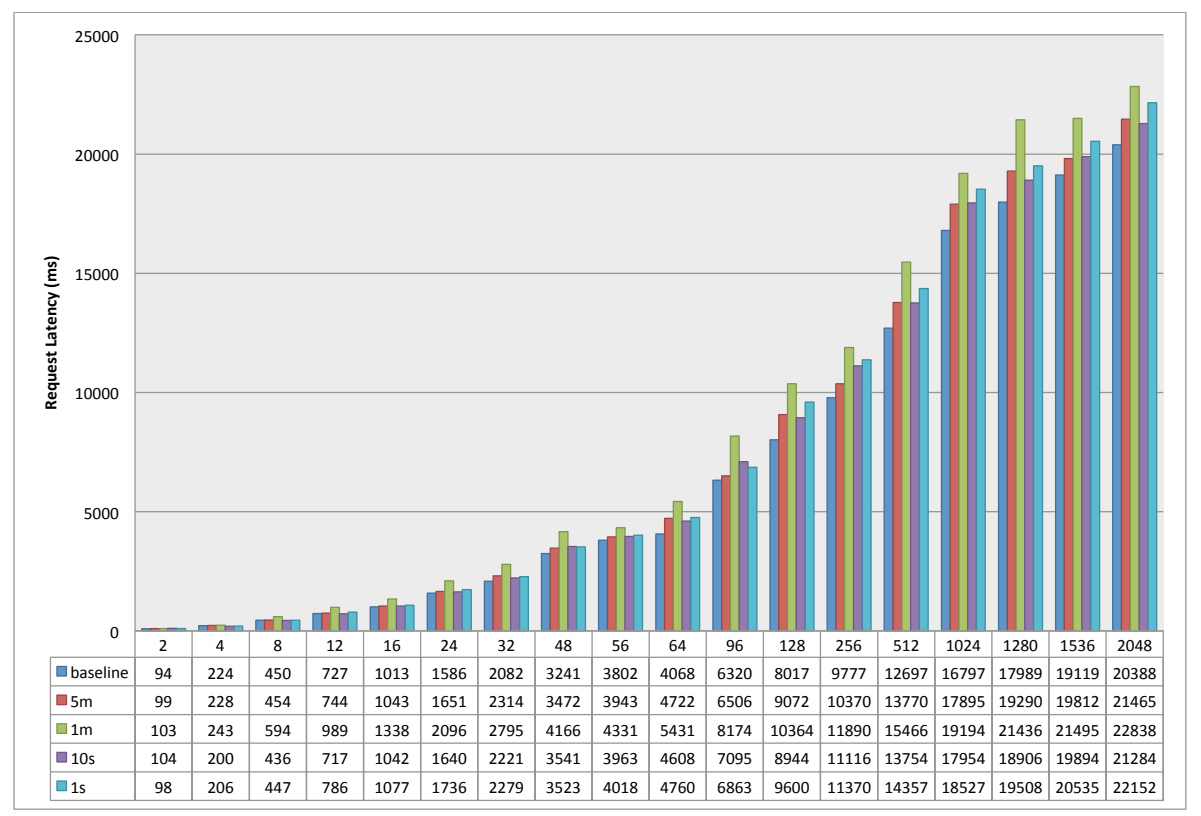

Figure 4.2 - Transparency - 25th Fibonacci

Apache's IO-bound performance measured in the 5MB download test is relatively unaffected by $d C A M P$. This is expected since the infrequent samples being logged to an output file are $d C A M P$ 's only disk access and the Data Protocol is designed to have a small network footprint. This graph also shows the 512 thread load point as the beginning of a trend line shift, again correlating with the increase in request error rate. 


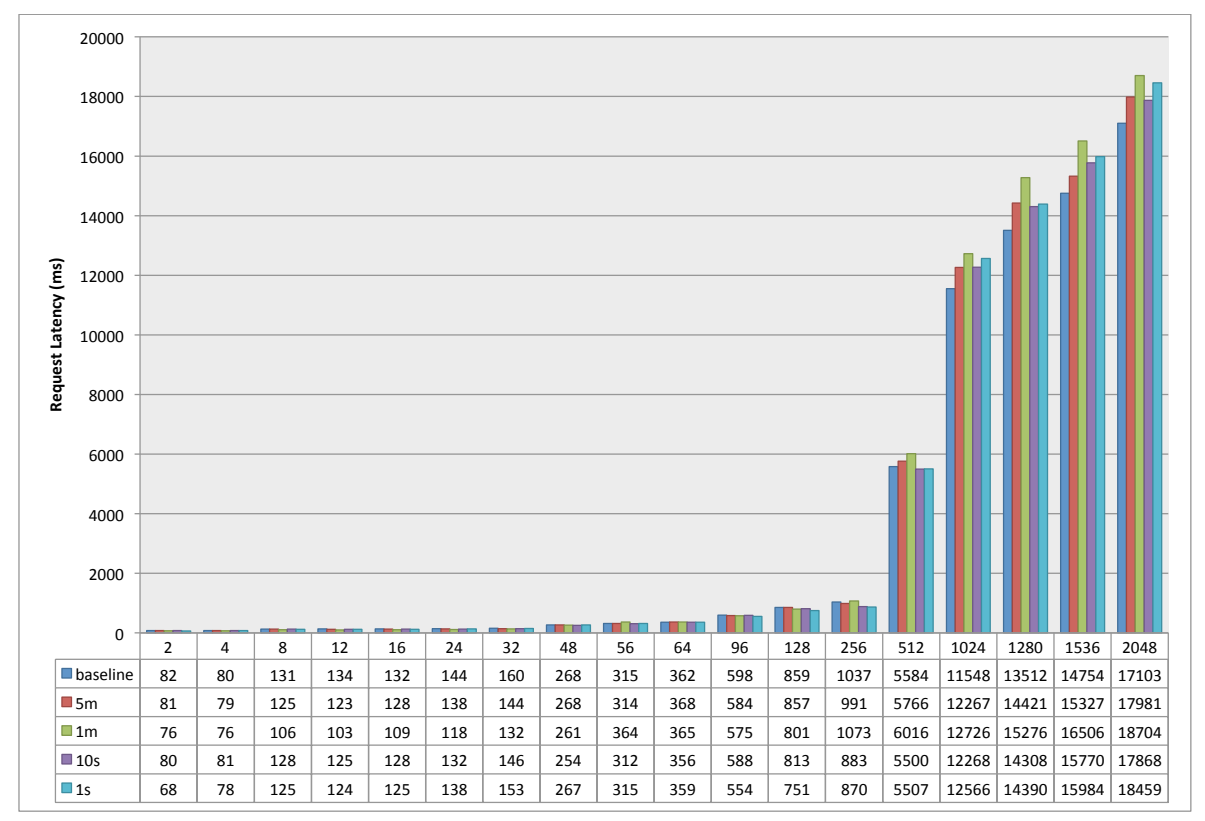

Figure 4.3 - Transparency - 5MB Download

A few conclusions can be drawn from these results.

When nodes are not expected to fail frequently, using longer heartbeat periods reduces the impact $d C A M P$ has on the system. It is better to monitor a process using a faster sample period than an entire system using a slower sample period. The $d C A M P$ system impact is noticeable but a considerably smaller factor than the impact hardware limitations have on performance monitoring.

Lastly, holding all else constant, slower sample periods have an obviously lower impact on system performance compared to faster sample periods. Possibly using $d C A M P^{\text {'s }}$ reporting threshold, system impact can be minimized while still maintaining fine sample granularity. 


\subsection{Scalability}

One of the primary measures of scalability for a distributed system is its network traffic.[30] By simulating successively larger $d C A M P$ systems (with respect to node count), one can extrapolate $d C A M P$ 's effectiveness at monitoring large distributed systems and how to best configure its metric collections.

\subsubsection{Workload}

$d C A M P$ is setup to monitor a machine's global metrics, scaling the number of simulated nodes in the $d C A M P$ system from three nodes (one Root, one Collector, one Metric) up to 200 nodes (eight groups with twenty-five nodes per group). The metric configuration is kept constant for each test run. As $d C A M P$ starts, monitors in steady state, and shuts down, the machine's network traffic is monitored and recorded every five seconds.

The test machine is a MacBook Pro (2.7GHz Core i7, 8GB 1333MHz DDR3, SSD) running OSX 10.9. All simulated $d C A M P$ nodes use endpoints on the machine's loopback interface, and only the loopback interface traffic is monitored. The machine is otherwise entirely idle during the test runs.

\subsection{2 dCAMP Configuration}

$d C A M P$ is configured to monitor and report the below global metrics, using a heartbeat of 60 seconds.

- CPU usage every 60 seconds

- total disk throughput every 120 seconds

- total network throughput every 120 seconds 
- memory usage every 60 seconds

No thresholds were defined for the above configuration. That is, Metric nodes immediately reported every sample instead of holding them for later reporting.

\subsubsection{Results}

Sparklines of each load point (not shown in this work) display the same pattern: highest network traffic occurs during start up and then also on shutdown. This pattern follows the design of $d C A M P$ which uses a chatty configuration protocol and a terse data protocol. The rest of steady-state operation shows an expected bursty pattern, with mostly low network traffic except on sample periods.

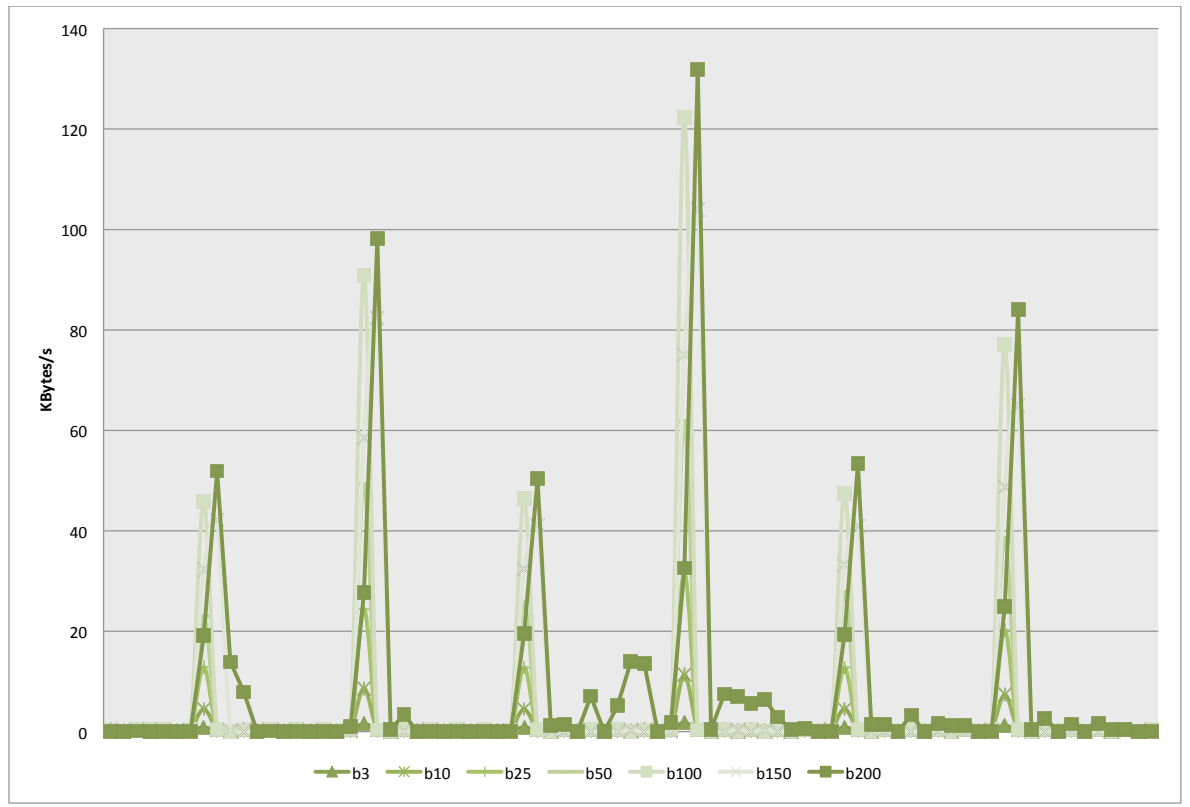

Figure 4.4 - Scalability - Steady-State Network Bytes: Network bytes during steady-state operation as the number of $d C A M P$ nodes increases. 


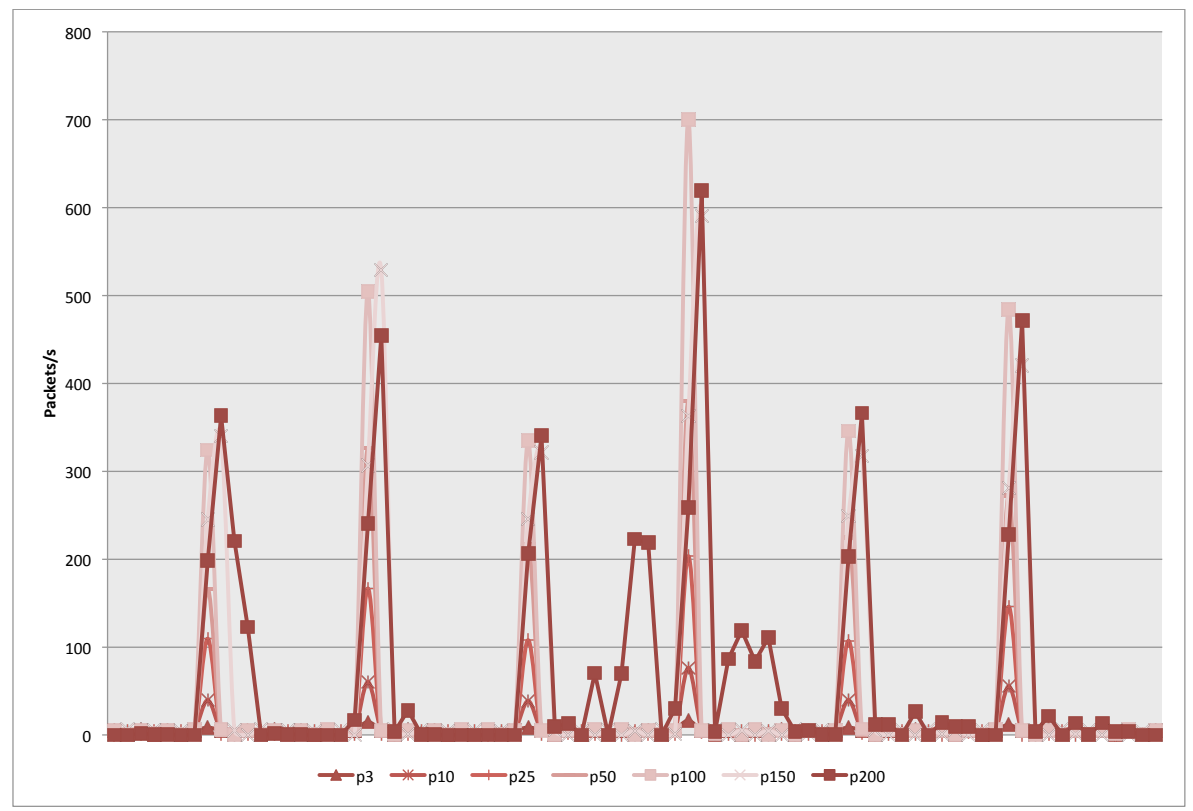

Figure 4.5 - Scalability - Steady-State Network Packets: Network packets during steady-state operation as the number of $d C A M P$ nodes increases.

As the node count increases, the rate at which bytes/packets are sent and received increases. This correlates with the larger configuration which $d C A M P$ must track as well as the additional nodes sending and receiving data. Looking at the same values but also relating them to the number of nodes in the system, one sees the configuration size grows faster than the number of nodes.

However, the number of messages being sent per node actually goes down and levels off just under 1 packet per node per second. This can be attributed to the fact that the number of Metric nodes increases faster in relation to the number of Collector nodes. That is, Metric nodes do not require full-configuration replication and send/receive fewer messages since they are relatively uninvolved with topology coordination in comparison to Collector nodes.

As this ratio increases, it is expected the number of messages per node to 
decrease. This latter observation indicates a higher number of child nodes per parent would result in lower network utilization and better $d C A M P$ scalability.

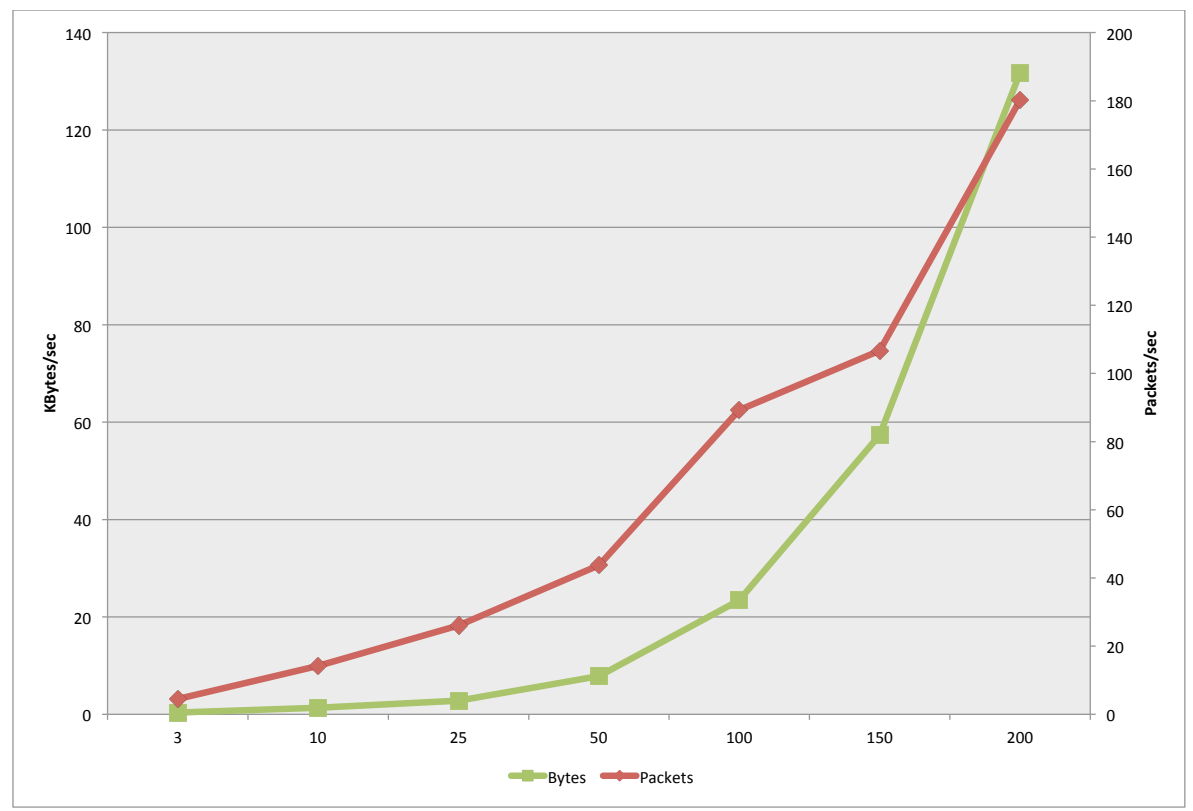

Figure 4.6 - Scalability - Average Network Utilization: Average network utilization as the number of $d C A M P$ nodes increases. 


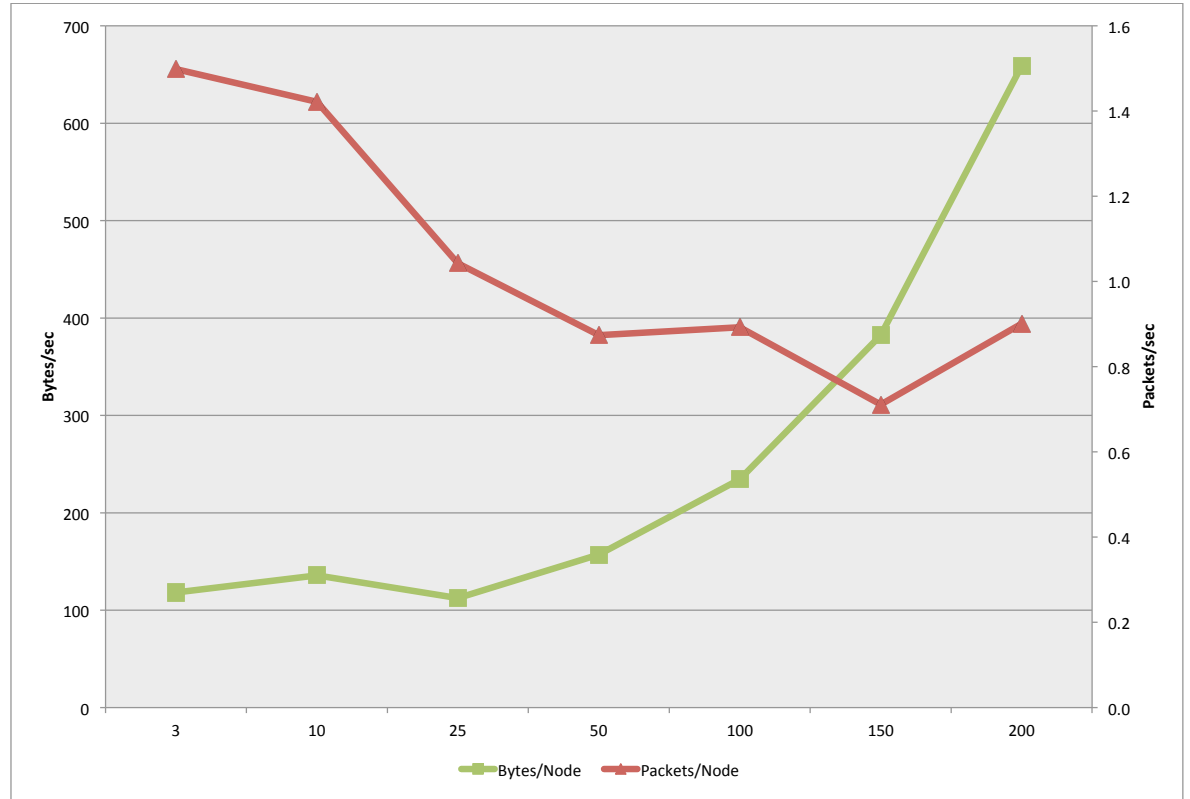

Figure 4.7 - Scalability - Average Network Utilization Per Node: Average network utilization per node as the number of $d C A M P$ nodes increases. 


\section{CHAPTER 5}

\section{Related Work}

Being distributed, a framework must collect data from a large number of nodes and aggregate the data to one node or client. Implementations have been built using centralized, hierarchical, peer-to-peer and any number of other architectures. There are three types of metric gathering techniques: (1) hardware counters and sensors use specialized hardware to gather highly accurate metrics and are highly dependent on the underlying hardware architecture, (2) software sensors use modern operating system interfaces to acquire moderately accurate performance metrics in an architecture-independent interface, and (3) hybrid approaches use a combination of hardware and software sensors to attain a balance between the two.

\subsection{Analysis}

There are a number of distributed performance frameworks being actively researched and developed, both academically and commercially. The frameworks listed in this section were chosen based on their categorization in the [30] taxonomy; only level 2 frameworks are included. Level 2 frameworks are defined as having at least one type of republisher in addition to producers; these frameworks

usually distribute functionality across multiple hosts. [30] A limited analysis is conducted by reviewing the available literature, and further analysis (i.e., verifying scalability, transparency, and validity) is left as future work. 


\subsubsection{NetLogger}

Work done by Brian Tierney and Dan Gunter [26] [9] presents the Network Application Logger Toolkit (NetLogger). This framework can be used to monitor the performance of distributed systems at a very detailed level. With a new logging format and activation service [10] the authors improved upon their previous work and increased the toolkit's scalability and data delivery models. NetLogger is being actively developed and is one of the more well known distributed performance frameworks. The toolkit is composed of four parts: an API and library for instrumenting a given application, a set of tools for collecting and sorting logs, performance sensors, and a visualization user interface for the log files.

Each part assumes the system clocks of the individual nodes are accurate and synchronized (the authors mention the use of NTP to achieve a required clock synchronization of one millisecond). The instrumentation of code allows NetLogger to gather more detailed data from an application-to-application communication path, such as traces of network packets through a call hierarchy. The instrumentation also allows the activation service to update the monitoring of

parts of the system dynamically as consumers subscribe to various events and metrics.

Their research has shown NetLogger to be highly scalable, complete, and transparent as well as valid. The activation service provides a push data delivery and can utilize the security mechanisms part of current web services in order to authenticate requests for performance data. NetLogger is currently implemented for $\mathrm{C}, \mathrm{C}++$, Java, Perl, and Python applications. Because the framework lacks black box characteristics, its portability is greatly reduced. 


\subsubsection{JAMM}

Java Agents for Monitoring and Management (JAMM) [25] is the fruit of work by the authors of NetLogger to build a monitoring system with managed sensors.

The JAMM system consists of six components: sensors, sensor managers, event gateways, directory service, event consumers, and event archives. There is a sensor manager on each host, with the sensors acting as producers for the gateways which they publish the data to. The gateways can then filter and aggregate the incoming data according to consumer queries. The directory service is used to publish the location of the sensors and gateways, allowing for dynamic discovery of active sensors by the consumers. The event archive is used for historical analysis purposes.

JAMM explicitly uses a pull data delivery model where data is only sent when requested by a consumer. The overall architecture is generally distributed with the directory service being centralized. JAMM, being heavily based off of NetLogger, inherits the validity, completeness, security, and transparency of NetLogger along with its lack of portability. JAMM does, however, prove itself in terms of scalability with it's own architecture.

\subsubsection{Hawkeye}

Hawkeye [11] is a monitoring and management tool for distributed systems which makes use of technology previously researched and developed as part of the Condor project [16]. Condor provides mechanisms for collecting information about

large distributed computer systems. Hawkeye is being readily developed and is freely available for download on Linux and Solaris. 
Hawkeye uses a general push delivery model by configuring Condor to execute programs, or modules, at given time intervals, collect performance data, and send it to the central manager. These modules are configurable such that the "period" of module execution can be set to a given time frame in seconds, minutes, or hours or the module can be executed in "continuous" mode where the module's execution never ends. The available modules for monitoring a Condor pool include: disk space, memory used, network errors, open files, CPU monitoring, system load, users, Condor Node, Condor Pool, and Grid Probe. Custom modules can also be developed and installed for monitoring of arbitrary resources and metrics. Data can be accessed from the central manager via an API, CLI, or GUI.

While no experiments have been run, the generally centralized manager reduces the Hawkeye framework's scalability, and its transparency is unknown. The frameworks module based producer architecture gives it an infinite completeness, but being only available on Linux and Solaris makes the framework less portable. Lastly, the ability to run jobs securely on target machines has been left as future work by the authors.

\subsubsection{SCALEA-G}

Truong and Fahringer present SCALEA-G [29], an unified monitoring and performance analysis system for distributed systems. It is based on the Open Grid Service Architecture [3] and allows for a number of services to monitor both grid resources and grid application. SCALEA-G uses dynamic instrumentation to profile and trace Java and $\mathrm{C} / \mathrm{C}++$ applications in both push and pull data delivery models, making the framework both scalable and portable. 
The SCALEA-G framework is composed of several services: directory service, archival service, sensor manager service, instrumentation service, client service, and user portal. These services provide the following functionality respectively: publishing and searching of producers and consumers, storage of performance results, management of sensors, dynamic instrumentation of source code, administering clients and analyzing data, and on-line monitoring and performance analysis.

The framework makes use of secure sockets to achieve secure communications and achieves high completeness via code instrumentation. Unfortunately, the authors do not provide any report on SCALEA-G's validity or transparency.

\subsubsection{IMPuLSE}

Integrated Monitoring and Profiling for Large Scale Environments [1] was designed to address "operating system-induced performance anomalies" and provide "accurate, low-overhead, whole-system monitoring." The authors have chosen to develop a message-centric approach which associates data with messages rather than hosts and a system-wide statistical sampling to increases the framework's scalability.

The IMPuLSE framework is still in the design stage, and therefore lacks any implementation data outside of their new message-centric design pattern which shows promising results. Unfortunately, this leaves the framework with unknown transparency, security, completeness, portability, and validity.

\subsubsection{Host sFlow}

sFlow is a network monitoring protocol consisting of sFlow Agents, built directly into the router and switch network device management layer by each vendor, 
which analyze traffic and send metrics to sFlow Collectors on the network. [23] Host sFlow is an open-source implementation of the sFlow protocol which uses sFlow Agents to monitor multi-vendor physical and virtual servers. Host sFlow is capable of application layer monitoring (e.g. node.js, Memcached), as well, and may be implemented directly by device/OS manufacturers for easier deployment. $[14]$

In supporting host and application performance metric analysis alongside network metrics in one common system, sFlow has an advantage over more traditional host-only distributed performance frameworks. While sFlow's claims to scalable and accurate network level monitoring have been validated, less work has been done to show the same for Host sFlow.

\subsubsection{Ganglia}

Ganglia is a distributed performance framework designed specifically for highperformance computing (HPC) environments, and it has been used to monitor real-world HPC, grid, and "planetary-scale" systems. Ganglia uses different protocols for intra- and inter-cluster communication: a multicast listen/announce protocol within a single cluster and a tree of point-to-point connections between clusters. Ganglia is well used and is actively used to monitor over 500 different systems. [17]

The analysis presented in [17] shows the design scales and maintains transparency for systems of several hundred nodes. Still, scalability is a concern of the authors since the multicast protocol exhibits a quadratic trendline as the number of nodes within a cluster increases. Memory usage and inter-cluster bandwidth also increase as the number of nodes increases, albeit much more linearly. In 
comparison, $d C A M P$ memory usage is nearly constant since performance data is not persisted in memory to the same extent.

\subsection{Summary}

There are a number of high quality and effective distributed performance frameworks being actively researched and developed, but with some frameworks having more research than others, there is a natural disparity of information about each framework. While the frameworks vary in distributed architecture and features, they all fulfill the minimum requirements of performance frameworks. The frameworks listed in this work are mainly software based sensor frameworks. This was chosen due to the inherent portability advantage of software sensors over hardware or hybrid sensors.

Many authors have failed to address their framework's validity, transparency, and scalability explicitly, thinking the framework's architecture speaks for itself or blindly assuming it is accurate and introduces negligible load on the measured system. It is left as future work to conduct formal experiments to test validity, transparency, and scalability of the distributed performance frameworks listed here. 


\title{
CHAPTER 6
}

\author{
Conclusions
}

This work presents $d C A M P$ : Distributed Common API for Measuring Performance, a distributed performance framework built on top of Mark Gabel and Michael Haungs' CAMP [4]. This work describes the design and implementation of $d C A M P$, using roles and services on top of ZeroMQ to build a simple, reliable distributed system.

A set of criterion for evaluating distributed performance frameworks is also given by extending and updating the criterion presented in [30]. This criterion is then used to evaluate $d C A M P$ along with several other related works.

\subsection{Summary of Contributions}

$d C A M P$ itself extends $C A M P$ with (1) a stateful performance measurement API, (2) distribution and aggregation of performance metrics, (3) filtering and triggering of performance metrics across the distributed system, and (4) simple fault tolerance to recover from node failures.

The updated distributed performance framework criterion is introduced and used to evaluate $d C A M P$. Chapter 4 presents an empirical evaluation of $d C A M P$ 's transparency and scalability. Validity and portability are inherited from $C A M P$ as well as the use of portable Python libraries. $d C A M P$ 's data delivery models and completeness are apparent in the system's design and configuration. 
Updating $d C A M P$ to meet the security criterion is unfinished work as described in the next section along with the rest of $d C A M P$ 's future directions.

\subsection{Future Work}

\subsubsection{Additional Features}

While $d C A M P$ in its current implementation meets the requirements of a basic $\mathrm{DPF}$, these features should advance it into a more complete, end-to-end distributed performance monitoring solution.

An end-to-end tool built on top of $d C A M P$ could allow a system administrator to quickly look at the performance of a large part of the network via aggregate metrics and easily drill down into the groups and/or nodes which exhibit problematic behaviour. Three options toward this goal are most readily apparent. The first would be to implement a lightweight web server within each Base node, adding support for REST API access to historical metric data along with a graphical user interface for easier $d C A M P$ system management. The second would be a more traditional API, allowing $d C A M P$ to run as a module inside another Python application. The third option is a slight variation of the second, exposing an API via ZeroMQ so $d C A M P$ continues to operate as a separate process but still gives direct programmatic access to performance data and system management.

The current $d C A M P$ protocols leave much to be desired when it comes to secure communication and operation, failing the Security criterion presented in Chapter 1. A more secure implementation would include a form of salted pass phrases with every control message or even encrypt all messages sent from one node to another. 
One of the possible pain points with $d C A M P$ is the control given to the system administrator through group specifications. Specifically, administrators are tasked not only with identifying which nodes to include in the system, but also how those nodes are placed into the distributed topology. Instead of this manual configuration, automatic grouping of nodes may be implemented based on network locality, metric configuration and sample periods, or even a tunable such as preference of network vs. CPU/memory overhead. The administrator would be left with the task of defining which metrics a given node should collect and $d C A M P$ would best select where the nodes sit in the hierarchy, how many children nodes a single parent manages, etc.

\subsubsection{Fault Tolerance}

The fault tolerance of $d C A M P$ could be improved by implementing these features which were considered out-of-scope for the original project.

$d C A M P$ does not support any fault tolerance for network failures - it only attempts to recover from node failures. It is assumed that if (part of) the network goes down, the lack of data from that subnet will suffice. Specifically, $d C A M P$ cannot currently tolerate a split-brain syndrome in which the network has been partitioned and entire subsets of the system cannot communicate with each other. It may be enhanced to recover from such network partitions, though.

The system time among multiple nodes in the distributed system may vary significantly. $d C A M P$ is not meant to be a high-resolution system with respect to the ordering of performance data occurrences. It is assumed that the standard Network Time Protocol (NTP) provides sufficient time synchronization across all nodes in the system OR the precise ordering of performance events in the system is not required. 
To further increase fault tolerance of the topology, $d C A M P$ should be able to operate without a Root node. That is, the Management service should not be continuously needed for the system to operate. Essentially this comes down to all top-level Collector nodes being potential endpoints for end-user control, at which point it momentarily acts as a Root, sending out configuration updates.

Lastly, as described in Chapter 3, dCAMP could become more resilient to software failures by running Base nodes within a self-restarting executable. If the process crashes for any reason, it would automatically be restarted and join back into the network.

\subsubsection{Improve Performance and Scalability}

With several places for improvement, increasing the efficiency and performance of $d C A M P$ 's own implementation could make really large systems feasible.

The current implementation of each ZeroMQ protocol heavily relies on a common polling pattern. Not only does this waste thread resources waiting on socket connections, but the code becomes hard to maintain as well. An alternate solution to this polling is event-driven I/O. ZeroMQ supports this alternate messaging pattern via Facebook's Tornado IOLoop[27][28] and libev via gevent[7][8].

With IOLoop, it may be possible to use a single IO loop, hosted by the Base node, shared among all the active services. This reduces the number of idle threads per node, freeing valuable operating system resources and reducing $d C A M P$ 's processing overhead.

Although $d C A M P$ only uses classic TCP protocols for all communication, ZeroMQ does support multicast network protocols. Using multicast judiciously within $d C A M P$ could greatly reduce configuration costs and network traffic, for 
example in the Topology Protocols. For $d C A M P$ systems spanning multiple subnets, the use of multicast would require special network configurations or special ZeroMQ gateways for passing messages from one subnet to the next.

Multiple-level branches are not supported in the current implementation. That is, all Collector nodes have the Root node as their parent and only have Metric nodes as their children. Extending support for multiple levels of Collectors would allow large group configurations to be automatically split into multiple (identically configured) branches for improved scalability

Compiling the various critical paths within $d C A M P$, such as the metric sampling code in the Sensor service, using Pyrex[19] or Cython[2] may boost performance and lower the cost of metric collection such that faster sample periods can be used without issue.

Due to Python's Global Interpreter Lock[21], there are limitations to the parallel execution of threads on an SMP system. While $d C A M P$ 's use of threads is heavily I/O-bound, some gains may also be found by using full-fledged processes instead of threads.

While not a huge cost, $d C A M P$ currently requires two nodes to execute alongside each other on a system which hosts a Collector. An improvement would be to provide full support for metric sampling directly within the Collector role.

\subsubsection{Metric Extensions}

Only a small subset of metrics were implemented in $d C A M P$ as a proof of concept. The rest of the full set listed in the $d C A M P$ Metrics section are left as future work. 
Beyond the list of statically defined metrics, user-defined metrics would expand the performance monitoring infinitely. This could be implemented as a Python module integrated into the distributed system being monitored or through a plug-in system built into $d C A M P$ itself.

Additionally, $d C A M P$ could support additional data types such as histograms and variable length strings or even more fine grained control over when metrics are sampled. For example, metrics could be collected on demand, driven by user requests via the Management service, or collected at a special "once" sample period so data is sent to the Root node only at start.

There are also two features which can be implemented to improve collection and reporting efficiency. First, a more compact data message format could be used to combine multiple data samples into a single message, e.g. for aggregation purposes or representing entire branches in the topology. This would improve network efficiency as fewer packets would require routing and data could be more effectively compressed. Second, metrics could be sampled regularly but reported randomly within the period in order to distribute arrival of data from child nodes and not overload the Aggregation service.

Lastly, $d C A M P$ could be extended to support some hardware performance counters, bringing it more in-line with hybrid performance frameworks. In particular, it would be interesting to add support for Graphical Processing Unit metrics such as those available via the NVIDIA Management Library[18] which already has Python bindings support [20]. 


\section{BIBLIOGRAPHY}

[1] P. G. Bridges and A. B. MacCabe. Impulse: integrated monitoring and profiling for large-scale environments. In $L C R$ '04: Proceedings of the 7th workshop on Workshop on languages, compilers, and run-time support for scalable systems, pages 1-5, New York, NY, USA, 2004. ACM.

[2] Cython: C-extensions for python. http://www. cython.org.

[3] I. Foster, C. Kesselman, J. M. Nick, and S. Tuecke. Grid services for distributed system integration. Computer, 35(6):37-46, 2002.

[4] M. Gabel and M. Haungs. CAMP: a common API for measuring performance. In LISA'0\%: Proceedings of the 21st conference on Large Installation System Administration Conference, pages 1-14, Berkeley, CA, USA, 2007. USENIX Association.

[5] H. Garcia-Molina. Elections in a distributed computing system. IEEE Transactions on Computers, 31(1):48-59, 1982.

[6] D. Garlan and M. Shaw. An introduction to software architecture. Technical report, Pittsburgh, PA, USA, 1994.

[7] gevent: A coroutine-based network library for python. http://www . gevent. org.

[8] libev: A full-featured and high-performance event loop. http://libev. schmorp.de. 
[9] D. Gunter, B. Tierney, B. Crowley, M. Holding, and J. Lee. Netlogger: A toolkit for distributed system performance analysis. International Symposium on Modeling, Analysis, and Simulation of Computer Systems, 0:267, 2000.

[10] D. Gunter, B. Tierney, K. Jackson, J. Lee, and M. Stoufer. Dynamic monitoring of high-performance distributed applications. In Applications, Proceedings of the 11th IEEE Symposium on High Performance Distributed Computing, pages 163-170, 2002.

[11] Hawkeye. http://www.cs.wisc.edu/condor/hawkeye/.

[12] P. Hintjens. Clustered hashmap protocol. http://rfc.zeromq.org/spec: 12/CHP, April 2011.

[13] P. Hintjens. Code Connected Volume 1, Learning ZeroMQ. CreateSpace Independent Publishing Platform, January 2013. http://zguide.zeromq. org.

[14] About Host sFlow: Data center wide server performance monitoring. http: //host-sflow. sourceforge.net/about.php.

[15] Apache JMeter. http://jmeter.apache.org.

[16] M. Litzkow, M. Livny, and M. Mutka. Condor-a hunter of idle workstations. Distributed Computing Systems, 1988., 8th International Conference on, pages 104-111, Jun 1988.

[17] M. L. Massie, B. N. Chun, and D. E. Culler. The ganglia distributed monitoring system: Design, implementation and experience. Parallel Computing, 30:817-840, 2003. 
[18] NVIDIA Management Library. https://developer.nvidia.com/ nvidia-management-library-nvml.

[19] Pyrex - a language for writing python extension modules. http://www. cosc.canterbury.ac.nz/greg.ewing/python/Pyrex/.

[20] Python bindings for the NVIDIA Management Library. https://pypi. python.org/pypi/nvidia-ml-py/.

[21] Python 3: Global interpreter lock. https://docs.python.org/3/ glossary.html\#term-global-interpreter-lock.

[22] R. Schaller. Moore's law: past, present and future. Spectrum, IEEE, 34(6):52-59, Jun 1997.

[23] Traffic monitoring using sFlow. http://www.sflow.org/sFlowOverview. pdf.

[24] M. Sustrik, M. Lucina, and P. Hintjens. zmq_socket - create ØMQ socket. http://api.zeromq.org/3-2:zmq-socket, 2012.

[25] B. Tierney, B. Crowley, D. Gunter, J. Lee, and M. Thompson. A monitoring sensor management system for grid environments. In Proceedings of the IEEE High Performance Distributed Computing Conference (HPDC-9), pages 97$104,2000$.

[26] B. Tierney, W. Johnston, B. Crowley, G. Hoo, C. Brooks, and D. Gunter. The netlogger methodology for high performance distributed systems performance analysis. In In Proc. 7th IEEE Symp. on High Performance Distributed Computing, pages 260-267, 1998.

[27] Tornado. http://www.tornadoweb.org. 
[28] tornado.ioloop - main event loop. http://www.tornadoweb.org/en/ stable/ioloop.html.

[29] H.-L. Truong and T. Fahringer. Scalea-g: A unified monitoring and performance analysis system for the grid. Sci. Program., 12(4):225-237, 2004.

[30] S. Zanikolas and R. Sakellariou. A taxonomy of grid monitoring systems. Future Gener. Comput. Syst., 21(1):163-188, 2005. 


\title{
APPENDIX A
}

\author{
ZeroMQ Primer
}

\section{A.1 Why ZeroMQ}

Not surprisingly, the most succinct description of ZeroMQ is found in The Guide[13] preface,

ØMQ (also known as ZeroMQ, OMQ, or zmq) looks like an embeddable networking library but acts like a concurrency framework. It gives you sockets that carry atomic messages across various transports like in-process, inter-process, TCP, and multicast. You can connect sockets N-to-N with patterns like fan-out, pub-sub, task distribution, and request-reply. It's fast enough to be the fabric for clustered products. Its asynchronous I/O model gives you scalable multicore applications, built as asynchronous message-processing tasks. It has a score of language APIs and runs on most operating systems. ØMQ is from iMatix [http://www.imatix.com] and is LGPLv3 open source.

No appendix could justly explain ZeroMQ or give the reader a true understanding of its abilities and proper use. Read The Guide (a freer and more up-to-date version is online at http://zguide.zeromq.org) and, if truly adventurous (or just morbidly curious), go through all $750+$ examples in any of the 28 programming languages available.

Forget about RPC, MPI, and raw sockets. ZeroMQ allows a developer to build distributed systems by focusing on the data and implementing simple design patterns. In short, ZeroMQ allows the distributed systems developer to have fun. No joke. 


\section{A.2 Sockets and Message Patterns}

To begin understanding ZeroMQ, a foundational knowledge of ØMQ sockets, messages, and patterns is needed.

\section{A.2.1 Sockets and Messages}

$\varnothing \mathrm{MQ}$ sockets mimic standard TCP sockets, exposing interfaces for creating and destroying instances, binding and connecting to network endpoints, and sending and receiving data. However, they have two key differences from their TCP counterparts.

First, they are asynchronous - the actual sending and receiving of data on a ZeroMQ socket is handled by a background thread. Second, ØMQ sockets have built-in support for one-to-many connections. That is, a single socket can send and receive data from multiple endpoints.

ZeroMQ sockets are explicitly typed, with the type dictating how data is routed and queued to and from the socket. Furthermore, this explicit typing means only certain socket types can be connected to each other.

ZeroMQ messages are the building blocks of all data sent across ZeroMQ sockets. A message is comprised of one or more frames (or parts), and a single frame can be any size (including zero) that fits in memory. ZeroMQ guarantees messages are delivered atomically, meaning either all frames of the message are sent/received or none of the frames. Lastly, because sockets are asynchronous and messages are atomic, the entire message must fit in memory. 


\section{A.2.2 Messaging Patterns}

Generally speaking, the ZeroMQ messaging patterns are defined by the socket routing and queuing rules as well as each socket's compatible type pairings. As listed in the zmq_socket man page[24], ZeroMQ supports the following core messaging patterns.

Publish-Subscribe: "The publish-subscribe pattern is used for one-to-many distribution of data from a single publisher to multiple subscribers in a fan out fashion."

The two socket types used for this pattern are PUB, which can only send messages, and SUB, which can only receive messages. Naturally, this is a unidirectional pattern. This work refers to the publish-subscribe pattern as $\mathrm{PUB} / \mathrm{SUB}$.

Request-Reply: "The request-reply pattern is used for sending requests from a $[\ldots]$ client to one or more $[. .$.$] services, and receiving subsequent replies$ to each request sent."

The two basic socket types for this pattern, REQ and REP, require strict ordering of messages: a message must be first be sent on the REQ socket before a message can be received on the socket, and vice versa for the REP socket. Two advanced socket types, XREQ (or DEALER) and XREP (or ROUTER), allow a more lenient communication pattern. This work refers to both request-reply patterns as REQ/REP.

Pipeline: "The pipeline pattern is used for distributing data to nodes arranged in a pipeline. Data always flows down the pipeline, and each stage of the pipeline is connected to at least one node. When a pipeline stage is connected to multiple nodes data is round-robined among all connected nodes." 
PUSH (send-only) and PULL (receive-only) socket types are used for this pattern. Like PUB/SUB, this is a unidirectional pattern. This work refers to the pipeline pattern as PUSH/PULL.

Exclusive Pair: "The exclusive pair pattern is used to connect a peer to precisely one other peer. This pattern is used for inter-thread communication across the inproc transport."

Only the PAIR socket type can be used for this pattern.

\section{A.3 Useful Features for $d C A M P$}

Apart from the general happiness ZeroMQ offers to the distributed systems developer, some features are particularly useful in $d C A M P$.

\section{A.3.1 Topic Filtering}

All messages sent using PUB/SUB are filtered (usually by the publisher) based on the "topics" to which each subscriber subscribes. Topics, stated simply, are the leading bytes of a message's first frame. By default, a SUB socket is not subscribed to any topics.

Consider a PUB message which contains b'/fruit/apple' as its first frame. A subscriber would receive this message if it subscribed to b'/fruit' or b'/' or even b' ' (an empty frame). But if the subscriber was not subscribed to any topics or only subscribed to the $\mathrm{b}^{\prime} / \mathrm{plants}$ ' topic, it would not receive the message. Do note: the topic can be any binary data, not just character data.

Topic filtering fits naturally into the Configuration Replication Protocol where different roles only replicate portions of the config and the Topology Discovery Protocol where the root node needs to send commands to a subset of the topology. 


\section{A.3.2 Easy Message Debugging}

ZeroMQ's atomic multipart message passing lends itself to what The Guide calls the "Cheap or Nasty pattern" [13]. That is, use cheap, easy to write/read, overly verbose messages for infrequent control scenarios and nasty, compact, highly performant messages for long-lived and frequent data scenarios.

In $d C A M P$, all control messages follow the cheap pattern, making them easy to debug. But the data messages, which tend to not need a lot of debugging, are free to be more optimized.

\section{A.3.3 Simplified Threading Design}

While much care must still be taken in their use, the inherent properties of ZeroMQ sockets (asynchronous nature, utilization of send/receive queues, ability to round-robin and fair-queue messages) allow for more attention to be paid to the design, purpose, and real work of each thread rather than the mechanics of sending and receiving data.

This is clearly seen in $d C A M P$ Service implementations where, for example, a single thread can be used to process both remote nodes' performance metrics and the local node's performance metrics without any special coding: one socket with multiple endpoints connected and the built-in fair-queuing taking care of routing.

\section{A.3.4 Quick Simulation}

The nature of ZMQ bind/connect endpoints just being a transport and a network address means simulation of large distributed systems can take place on a single 
machine using unique port numbers. Additionally, the interoperability of inproc (within the same process) and TCP transports allows for even larger simulations, not bound by port availability on the host.

This is demonstrated several times in examples within The Guide, and it indeed held true during $d C A M P$ development and testing. 


\title{
APPENDIX B
}

\author{
Real Life
}

Finishing a master's thesis after college is no joke. Get it done now. Seriously. Do not waste your time. Real life is full of real work. Real deadlines. Relationships. Marriage. Babies. Nothing slows down.

Life is not waiting for you to complete unfinished work. There will be a tinge of guilt with every new project and every day spent not working towards its completion. Goodwill runs out; good intentions are just false promises.

Plus, a degree is a nice thing to have, if not now, at least at some point in the future. Best of luck! 